\title{
Liver $X$ receptor $\alpha$ mediates hepatic triglyceride accumulation through upregulation of G0/G1 Switch Gene 2 expression
}

\author{
Bradlee L. Heckmann, ${ }^{1,2,3}$ Xiaodong Zhang, ${ }^{1,2}$ Alicia M. Saarinen, ${ }^{1,2}$ Gabriele Schoiswohl, ${ }^{4}$ \\ Erin E. Kershaw, ${ }^{4}$ Rudolf Zechner, ${ }^{5}$ and Jun Liu ${ }^{1,2,6}$ \\ 'Department of Biochemistry and Molecular Biology, ${ }^{2} \mathrm{HEAL}{ }^{\text {th }}$ Program, Mayo Clinic in Arizona, Scottsdale, Arizona, USA. \\ ${ }^{3}$ Mayo Graduate School, Rochester, Minnesota, USA. ${ }^{4}$ Division of Endocrinology, Department of Medicine, University of \\ Pittsburgh, Pittsburgh, Pennsylvania, USA. ${ }^{5}$ Institute of Molecular Biosciences, University of Graz, Graz, Austria. \\ ${ }^{6}$ Division of Endocrinology, Mayo Clinic in Arizona, Scottsdale, Arizona, USA.
}

Liver X receptors (LXRs) are transcription factors essential for cholesterol homeostasis and lipogenesis. LXR $\alpha$ has been implicated in regulating hepatic triglyceride (TC) accumulation upon both influx of adipose-derived fatty acids (FAs) during fasting and stimulation of de novo FA synthesis by chemical agonism of LXR. However, whether or not a convergent mechanism is employed to drive deposition of FAs from these 2 different sources in TGs is undetermined. Here, we report that the G0/G1 Switch Gene 2 (GOS2), a selective inhibitor of intracellular TC hydrolysis/ lipolysis, is a direct target gene of LXR $\alpha$. Transcriptional activation is conferred by LXR $\alpha$ binding to a direct repeat 4 (DR4) motif in the GOS2 promoter. While $L X R \alpha^{-1-}$ mice exhibited decreased hepatic GOS2 expression, adenoviral expression of GOS2 was sufficient to restore fasting-induced TC storage and glycogen depletion in the liver of these mice. In response to LXR agonist T0901317, COS2 ablation prevented hepatic steatosis and hypertriglyceridemia without affecting the beneficial effects on HDL. Thus, the LXR $\alpha$-COS2 axis plays a distinct role in regulating hepatic TC during both fasting and pharmacological activation of LXR.

Conflict of interest: The authors have declared that no conflict of interest exists.

Submitted: May 23, 2016

Accepted: January 9, 2017

Published: February 23, 2017

Reference information:

JCI Insight. 2017;2(4):e88735. https:// doi.org/10.1172/jici.insight.88735.

\section{Introduction}

The synthesis and hydrolysis of intrahepatic lipids are critical processes for the maintenance of systemic energy homeostasis (1-3). During the fed state, insulin stimulates storage of dietary fatty acids (FAs) in the form of triglycerides (TGs) in white adipose tissue (WAT). In contrast, fasting induces the efflux of free FAs from WAT and their subsequent uptake in nonadipose tissues, including liver. Free FAs taken up by hepatocytes can either be oxidized in mitochondria or reesterified into TGs, which are subsequently packaged and resecreted as very low-density lipoprotein (VLDL) particles. During fasting, the rate of hepatic FA uptake frequently exceeds the net rate of FA utilization, leading to increased TG synthesis and storage within intracellular lipid droplets (4-6).

Studies by our laboratory and others have identified G0/G1 Switch Gene 2 (G0S2) as a selective endogenous inhibitor of adipose triglyceride lipase (ATGL) (7-10), the rate-limiting TG hydrolase in various tissues including adipose tissue, cardiac muscle, and liver (11-16). During fasting, G0S2 is upregulated in the liver, where it acts critically to coordinate hepatic substrate utilization by decreasing the amount of available FAs and increasing the rates of glycogen breakdown (17-19). Specifically, global KO of G0S2 in mice leads to an impaired hepatic fasting response in terms of TG accumulation and glycogen depletion, along with enhanced adipose lipolysis (20-22). Mice with liver-specific G0S2 ablation exhibit increased hepatic ATGL-mediated TG hydrolysis and FA oxidation, along with decreased TG storage upon fasting (20). Despite the recent evidence that hepatic G0S2 expression is induced during fasting downstream of adipose lipolysis, as seen in mice lacking the lipase coactivator comparative gene identification-58 (CGI-58-ATko) (23), the identity of the responsible transcription factor in the liver is still unknown. 
The liver X receptor family of transcription factors ( $\mathrm{LXR} \alpha$ and $\operatorname{LXR} \beta)$ is known to play a central role in the regulation of cholesterol homeostasis and lipid synthesis $(24,25)$. LXRs control hepatic lipogenesis mainly through mediating the expression of SREBP-1c and its target genes for FA synthesis, including acetyl-CoA carboxylase (ACC), fatty acid synthase (FAS), and stearoyl-CoA desaturase-1 (SCD1) (2629). Accordingly, LXR $\alpha \beta$ double KO mice are characterized by markedly reduced hepatic FA synthesis and incorporation of FA into phospholipids and TGs $(30,31)$. Interestingly, deletion of LXR $\alpha$ alone also hampers the hepatic TG accumulation and adaptive response to fasting (32), suggesting a regulatory role of LXR $\alpha$ during the influx of adipose-derived FAs. Additionally, synthetic LXR agonists, albeit eliciting antiatherogenic activity via increasing tissue cholesterol efflux and reverse cholesterol trafficking through HDL, are considered undesirable as therapeutic agents due to their detrimental effects of causing hepatic steatosis and hypertriglyceridemia. Genetic studies have defined hepatic LXR $\alpha$ as the predominant subtype required for agonist-induced lipogenesis and formation of steatotic liver (33-35). As hepatic TG accumulation during fasting and in response to LXR agonism both require LXR $\alpha$, the question arises as to whether a convergent mechanism exists that promotes the deposition of FAs from different sources, adipose-derived versus endogenously generated, as exists for TGs in the liver.

In the present study, we have obtained evidence that hepatic G0S2 is a direct LXR $\alpha$ target gene during fasting and in response to pharmacological agonism of LXR. Consistent with this observation, we have discovered that the effect of LXR activation on liver TG content is mediated through enhanced G0S2 expression.

\section{Results}

Hepatic expression of GOS2 is upregulated by adipose-derived FAs. In WT mice, fasting increased hepatic mRNA expression of G0S2 by 8.3-fold and ATGL by 2.3-fold (Figure 1A), indicating a relatively greater effect on G0S2. To determine the contribution of adipocyte lipolysis to the fasting-induced upregulation in liver, we next compared their expression in adipocyte-specific ATGL KO (AAKO) mice that have previously been shown to have severely restricted adipocyte lipolysis (36). In the fasted AAKO mice, mRNA expression of G0S2 was 9.1-fold lower and ATGL was 6.58-fold higher than in the WT mice (Figure 1B). These data suggest that, during fasting, increased hepatic expression of G0S2 but not ATGL is dependent on adipose lipolysis, agreeing with what was recently observed in CGI-58-ATko animals (23).

To ascertain whether adipose lipolysis was directly responsible for activating G0S2 expression in hepatocytes, we cultured mouse primary hepatocytes in conditioned media collected from WT and ATGL ${ }^{-1}$ (ATGL KO) fat explants. The fat explants were pretreated with either vehicle alone or a $\beta 3$ receptor agonist CL316243 (CL) to stimulate adipocyte lipolysis. As shown in Figure 1C, expression of G0S2 in the primary hepatocytes was increased by 8.5 -fold when cultured in the media derived from CL- versus vehicle-treated WT fat explants. However, the effect of CL stimulation was largely abolished with the media derived from ATGL KO explants (Figure 1C). In comparison, no increase of ATGL expression was observed in the hepatocytes under all conditions. These data confirm that the output of stimulated adipose lipolysis directly drives hepatic expression of G0S2. Moreover, gas chromatographic analysis identified linoleic acid (18:2), oleic acid (18:1), palmitic acid (16:0), and palmitoleic acid (16:1) as the major FA species released from fat explants upon CL stimulation (Figure 1D). Interestingly, treatment with linoleic acid (18:2) or oleic acid (18:1) individually at concentrations consistent with the quantities released from the explants upon CL stimulation significantly increased expression of G0S2 in the primary hepatocytes (Figure 1E). The expression of ATGL was unaffected by individual FA treatment. Additionally, neither G0S2 nor ATGL was changed by treatment with glycerol (Supplemental Figure 1; supplemental material available online with this article; https://doi.org/10.1172/jci.insight.88735DS1), the other lipolytic product released from adipose tissue. These results demonstrate a direct effect of adipose-derived unsaturated FAs to upregulate G0S2 in hepatocytes.

Hepatic GOS2 expression in response to adipose lipolytic stimulation is dependent on LXR $\alpha$ but not PPAR . Since the phenotypes exhibited by LXR $\alpha$-deficient mice during fasting are closely reminiscent of those caused by G0S2 ablation $(20,32)$, we asked whether increased hepatic G0S2 expression in response to adipose-derived FAs would be related to LXR $\alpha$. Using qPCR and immunoblotting, we analyzed G0S2 expression in liver tissue isolated from WT and $\mathrm{LXR}^{-/-}(\mathrm{LXR} \mathrm{KO})$ mice. Compared with ad libitum feeding, fasting caused a robust increase in the hepatic G0S2 mRNA and protein levels in the WT animals (Figure 2, A and B). Expression of LXR $\alpha$ and 2 of its target genes ABCG5 and ABCA1 were also modestly but significantly increased upon fasting, while the expression of SREBP-1c was decreased. Strikingly, the induction of G0S2 along with ABCG5 
A

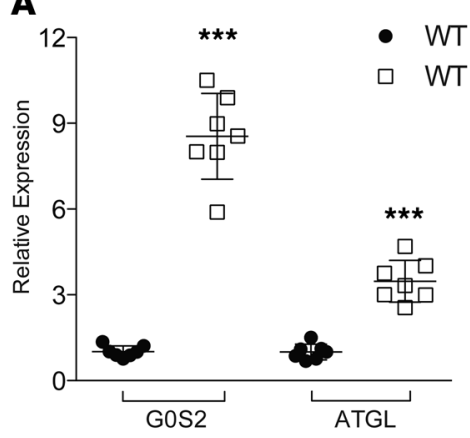

B

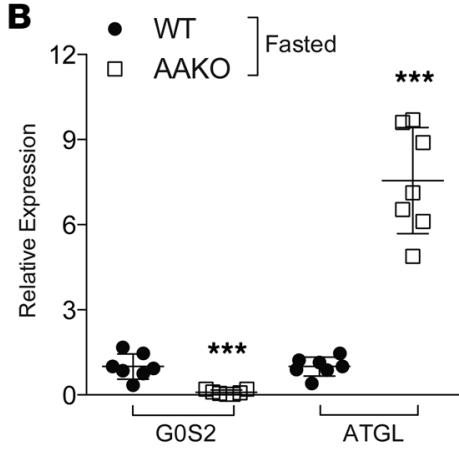

C

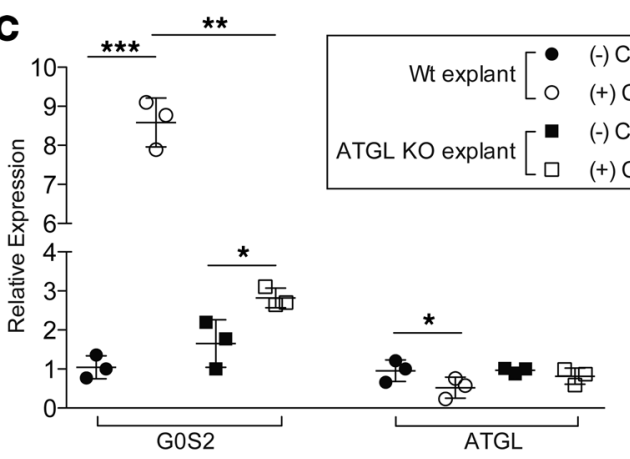

D

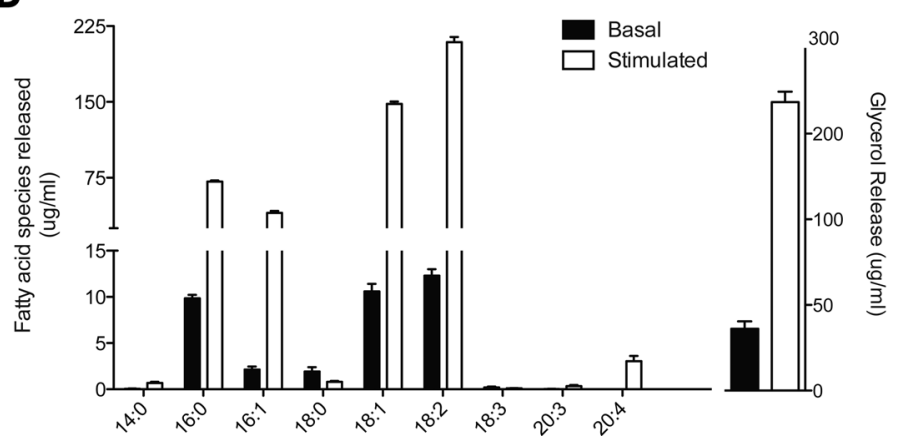

E

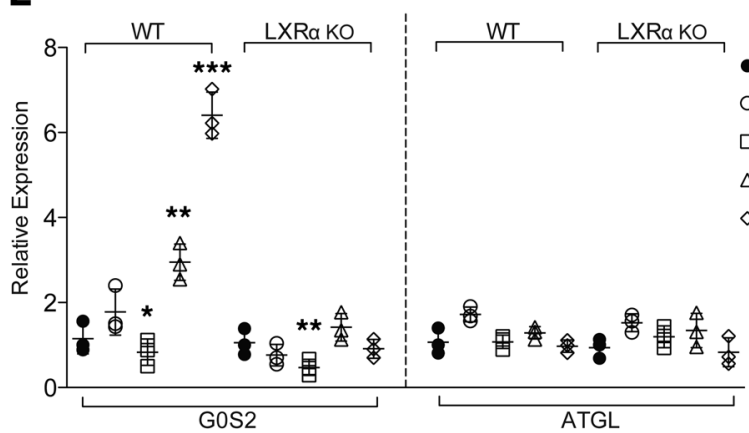

Figure 1. Hepatic GOS2 expression occurs in response to adipose-derived fatty acids. (A) qPCR analysis of hepatic GOS2 and ATCL mRNA expression in 14-week-old male WT mice either fed or fasted for 16 hours. (B) qPCR analysis of hepatic GOS2 and ATCL mRNA expression in 24-week-old male WT and adipose-specific ATCL KO mice (AAKO) mice fasted for 12 hours. (C) WT primary hepatocytes were cultured in adipose explant media as shown. qPCR analysis of GOS2 and ATCL expression in response to coculture in the presence or absence of lipolytically stimulated adipose explants was determined. (D) Adipose tissue isolated from 12-week-old female WT mice was explant cultured and lipolytically stimulated with CL316243 (CL). Media was collected, and fatty acid species released in response to lipolytic activation, along with glycerol released, were profiled and quantified, along with glycerol. (E) Major lipolytically released fatty acid species were individually conjugated to BSA at molar concentrations consistent with those released from adipose explants upon lipolytic stimulation and used to treat WT and LXR $\alpha$ KO primary hepatocytes. Concentrations are as follows: $C 16: 0=0.25$ $\mathrm{mM}, \mathrm{C} 16: 1=0.16 \mathrm{mM}, \mathrm{C} 18: 1=0.5 \mathrm{mM}$, and C18:2 $=0.725 \mathrm{mM}$. qPCR analysis of G0S2 and ATCL expression in response to fatty acid treatment is shown in panel $\mathbf{E}$. (For mice $n=7$; for cells $n=3$ for qPCR performed in triplicate; ${ }^{*} P<0.05,{ }^{* *} P<0.01,{ }^{* *} P<0.001$, Student's $t$ test.)

and ABCA1 in response to fasting was largely lost in the LXR KO mice (Figure 2, A and B). Hepatic ATGL expression, on the other hand, was increased during fasting regardless of the presence of LXR $\alpha$ (Figure 2, A and B). Similarly, acute stimulation of adipose lipolysis by CL injection increased hepatic G0S2 expression markedly in the WT mice (Figure 2, C and D). However, the LXR $\alpha$ expression was unaffected by CL. The induction of GOS2 expression was likewise abolished in the LXR KO mice. In comparison, ATGL expression exhibited a modest increase similarly in WT and LXR KO mice following CL injection (Figure 2, C and D). Taken together, these data indicate an LXR $\alpha$-dependent expression of G0S2 in the liver in response to both fasting- and CL-stimulated adipose lipolysis. Moreover, treatment with individual FAs failed to induce G0S2 expression in LXR KO primary hepatocytes (Figure 1E), confirming that the effect of adipose-derived FAs is mediated through LXR $\alpha$. Furthermore, LXR $\alpha$ is known to function in a heterodimer with RXR, a FA-responsive transcription factor. Interestingly, treatment of WT mice with a RXR antagonist HX531 also profoundly reduced hepatic expression of G0S2 mRNA and protein upon fasting or CL stimulation (Figure 2, E-H).

The heterodimeric transcription factor PPAR $\alpha /$ RXR plays a critical role in mediating fasting-induced adaptive energy response and hepatic FA oxidation. A previous study has shown a differential expression of G0S2 in the liver of PPAR $\alpha^{-1-}$ mice (37). However, treatment of mouse primary hepatocytes with a PPAR $\alpha$ agonist Wy14643 elicited no effect on G0S2 transcription (Figure 3A), while causing a considerable increase in the expression of PPAR $\alpha$ target genes, including AcadM, CPT1 $\alpha$, and AcotI. In addition, injection of mice with a PPAR $\alpha$ antagonist GW6471, though decreasing expression of PPAR $\alpha$, AcadM, CPT1 $\alpha$, and AcotI (Figure 3B), failed to inhibit fasting-induced expression of G0S2 mRNA and protein in liver (Figure 3, B and C). These results clearly demonstrate that PPAR $\alpha$ is not directly involved in mediating the induction of hepatic G0S2 expression. 
A

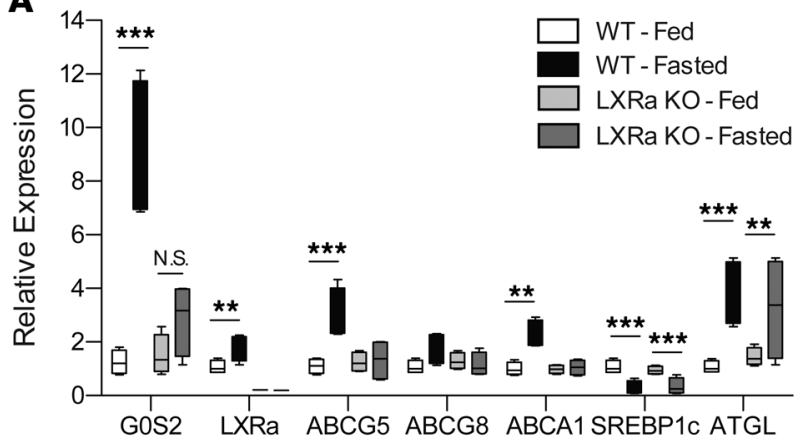

B

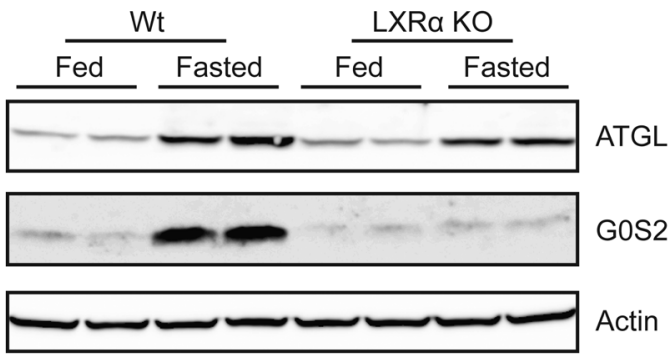

D

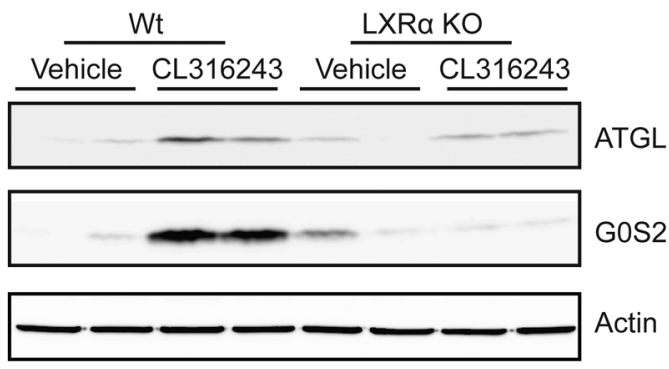

$\mathbf{F}$

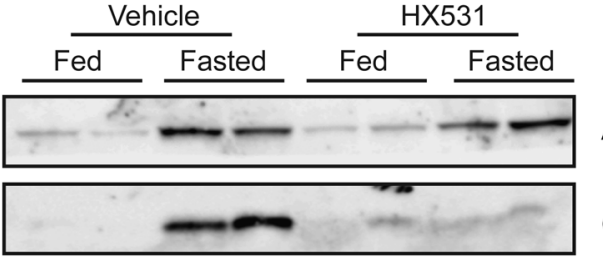

ATGL

GoS2

Actin

H

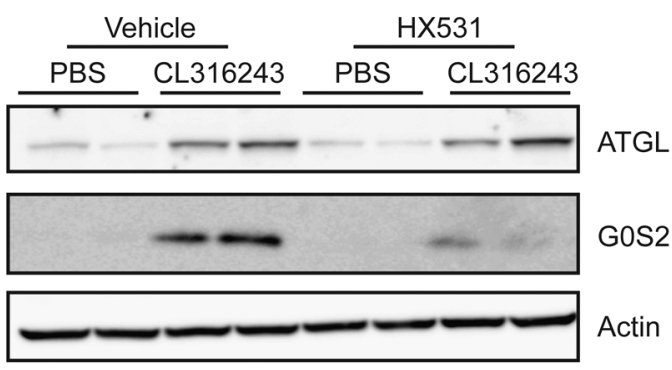

Figure 2. LXR $\alpha$ and RXR $\alpha$ are required for hepatic GOS2 expression in response to fasting and acute stimulation of adipose lipolysis. For panels $A$ and $B$, 12-week-old female WT and LXR $\alpha$ KO mice were fasted for 16 hours. (A) qPCR analysis of GOS2, ATCL, LXR $\alpha$, and LXR $\alpha$ target genes in liver. (B) Immunoblot analysis of GOS2 and ATCL expression in liver from 16-hour fasted WT and LXR $\alpha$ KO mice. For panels $\mathbf{C}$ and $\mathbf{D}, 14$-week-old female WT and LXR $\alpha$ KO mice were injected with $0.1 \mathrm{mg} / \mathrm{kg}$ CL316243 for 1 hour. (C) qPCR analysis of hepatic GOS2, ATCL, LXR $\alpha$, and LXR $\alpha$ target genes in response to CL injection. (D) Immunoblot analysis of GOS2 and ATCL expression in liver following acute CL injection in WT and LXR $\alpha$ KO mice. For panels $\mathbf{E}$ and $\mathbf{F}$, 14-week-old female WT mice were injected with vehicle or HX531 and then fasted for 16 hours. (E) qPCR analysis of hepatic GOS2 and ATCL expression. (F) Immunoblot analysis of GOS2 and ATCL expression in fasted liver. For panels $\mathbf{G}$ and $\mathbf{H}$, 15-week-old female WT mice were injected with CL316243 as described above in the presence or absence of HX531. (C) qPCR analysis of GOS2 and ATCL from liver. (H) Immunoblot analysis for hepatic G0S2 and ATCL in CL/HX531 mice. Box-and-whisker plots depict median (line within box), 25th percentile and 75th percentile (bottom and top borders), and range of minimum to maximum values (whiskers; $n=7$ [A and $\mathbf{C}], n=5$ [E and $\mathbf{G}]$ for qPCR performed in triplicate; ${ }^{*} P<0.05,{ }^{* *} P<0.01,{ }^{* *} P<0.001$, Student's $t$ test). 

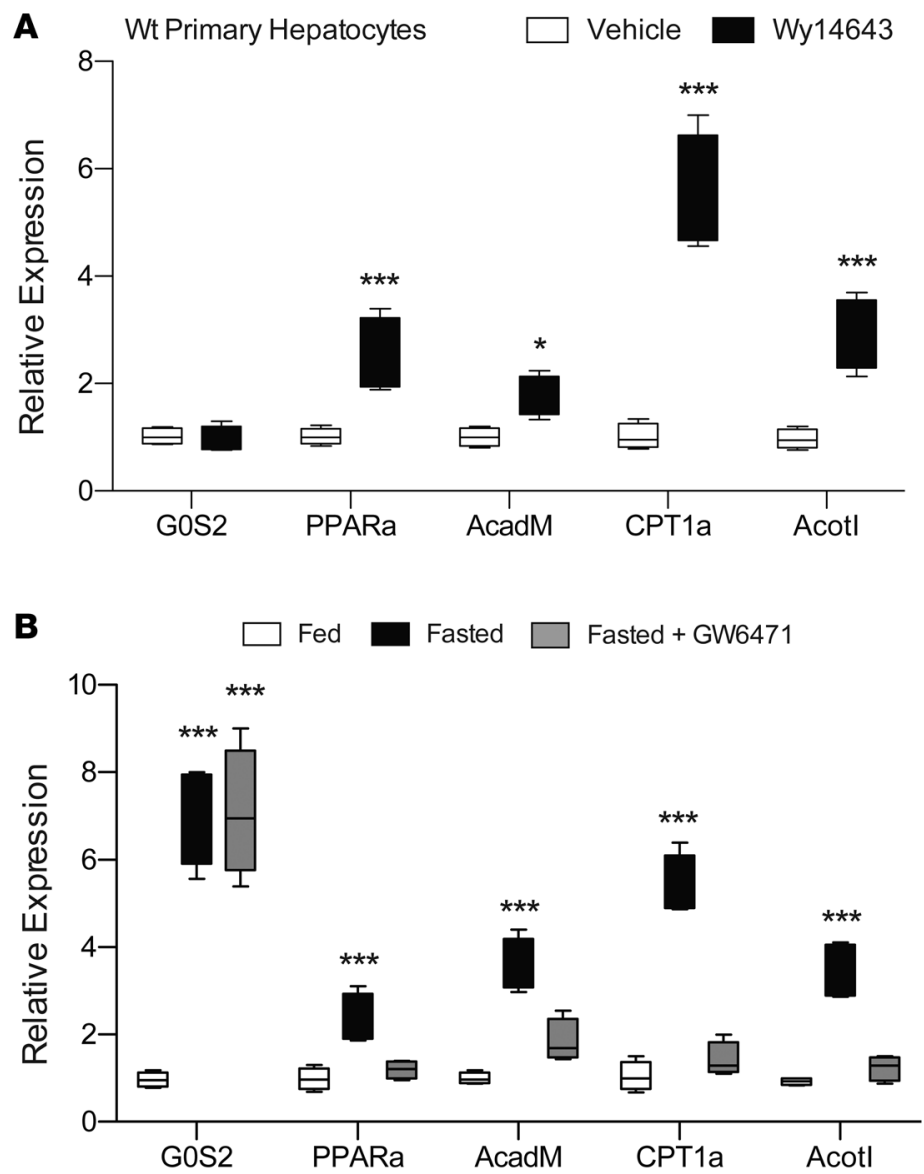

C
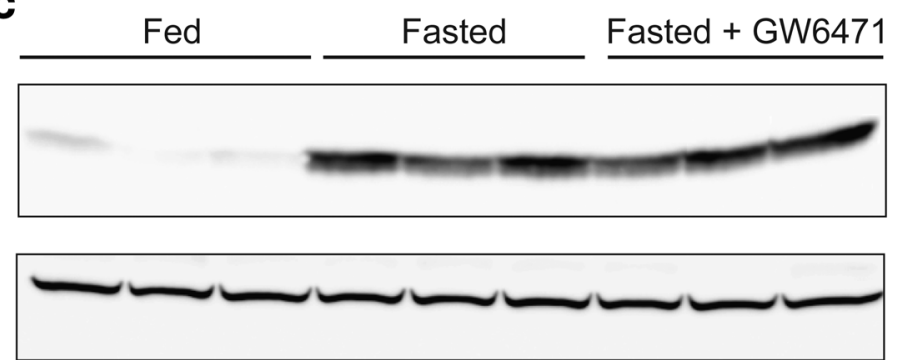

G0S2

Actin
Figure 3. Activation or inhibition of PPAR $\alpha$ does not impact COS2 expression. (A) qPCR analysis of GOS2, PPAR $\alpha$, and target genes from WT primary hepatocytes treated with 50 $\mu M$ Wy14643. (B) qPCR analysis of hepatic GOS2, PPAR $\alpha$, and PPAR $\alpha$ target genes from 14-week-old female WT mice fasted for 16 hours in the presence or absence of the PPAR $\alpha$ antagonist CW6471 at a dose of $1 \mathrm{mg} / \mathrm{kg}$. (C) Immunoblot analysis for COS2 expression. Box-and-whisker plots depict median (line within box), 25th percentile and 75th percentile (bottom and top borders), and range of minimum to maximum values (whiskers; $n=5$ per group; ${ }^{*} P<0.05,{ }^{* * *} P<0.0001$, Student's $t$ test).

$L X R \alpha$ mediates hepatic GOS2 expression in response to chemical agonism. To directly evaluate the effect of LXR activation on G0S2 expression, we injected WT mice with either T0901317 (T09) or GW3965, 2 synthetic LXR agonists. Upon LXR activation by T09, hepatic G0S2 mRNA expression was increased by over 7-fold, markedly exceeding that of known LXR target genes, including ABCG5, ABCG8, and SREBP1c (Figure 4A). Similar effects were observed with GW3965 injection (Supplemental Figure 2). Cotreatment of mice with either LXR antagonist GSK2033 or RXR antagonist HX531 significantly reduced the mRNA expression of G0S2 and other LXR targets induced by T09 (Figure $4 \mathrm{~A}$ ), again demonstrating the involvement of the LXR/ RXR heterodimer. To establish that increased G0S2 expression is caused by transcriptional activation and not due to enhanced mRNA stability, we took advantage of a unique feature of our G0S2 $2^{-/-}$(G0S2 KO) mice that have a $L a c Z$ reporter placed under the control of the endogenous G0S2 promoter (20). As indicated by $\beta$-galactosidase staining of liver sections from these mice, G0S2 transcription was robustly activated by LXR agonism (Figure 4B). More importantly, T09 injection failed to induce hepatic mRNA expression of G0S2, ABCG5, ABCG8, ABCA1, and SREBP-1c in LXR KO mice (Figure $4 \mathrm{C}$ ). In accordance with the gene transcription, the hepatic G0S2 protein levels also increased in the WT but not LXR KO mice in response to T09 (Fig-

ure 4D). In contrast to fasting and CL stimulation, T09 did not affect ATGL expression in the liver, regardless of LXR $\alpha$ (Figure 4, C and D). Together, these results demonstrate the LXR $\alpha$-dependent nature of G0S2 regulation, as well as argue against the regulation of ATGL by LXR $\alpha$. This differential regulation of G0S2 and ATGL by LXR $\alpha$ would be expected to alter the molar ratio of the enzyme and its inhibitor in hepatocytes, thereby affecting hepatic lipolysis.

LXR $\alpha$ mediates GOS2 transcription via direct promoter binding. A nuclear hormone receptor binding site (NHR) scan predicted that a putative LXR response element (LXRE) containing a direct repeat 4-like motif (DR4) is located $\sim 2 \mathrm{~kb}$ upstream of the transcriptional start codon of the human, rat, and mouse G0S2 genes (Figure 5A). To determine whether LXR $\alpha$ directly binds to this DR4 motif to mediate transcriptional activation in vivo, we performed ChIP assays on liver samples collected from fed and fasted mice. Relative to ad libitum feeding, a 16-hour fast induced an over 5-fold enrichment of LXR $\alpha$ at the putative LXRE sequence of the G0S2 promoter, while LXR $\alpha$ binding to the known LXRE sites of SREBP1c and ChREBP was drastically reduced upon fasting (Figure 5B). In comparison, LXR activation by T09 caused an over 15-fold enrichment of LXR $\alpha$ at the G0S2 LXRE (Figure 5C). By contrast, no LXR $\alpha$ binding was detected in the $5^{\prime}$ and $3^{\prime}$ regions flanking the putative G0S2 LXRE (Figure 5, B and C). Interestingly, T09 activation 
A

A

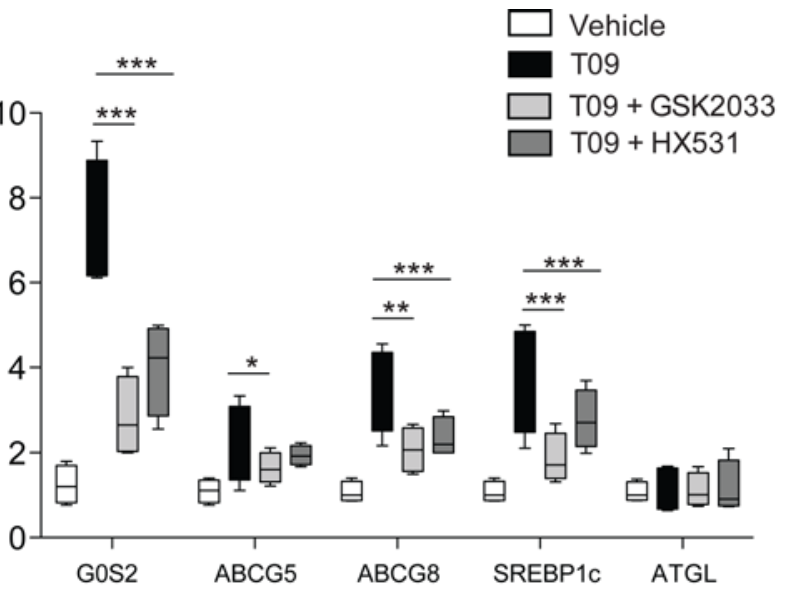

B

Vehicle

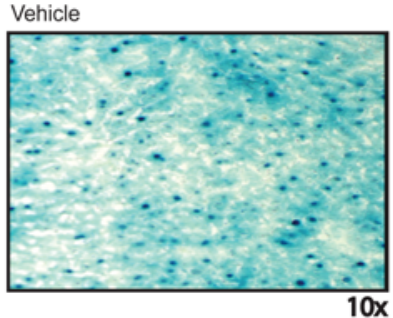

T09

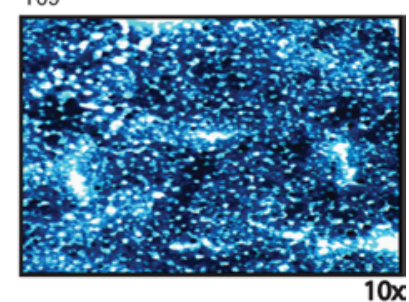

D
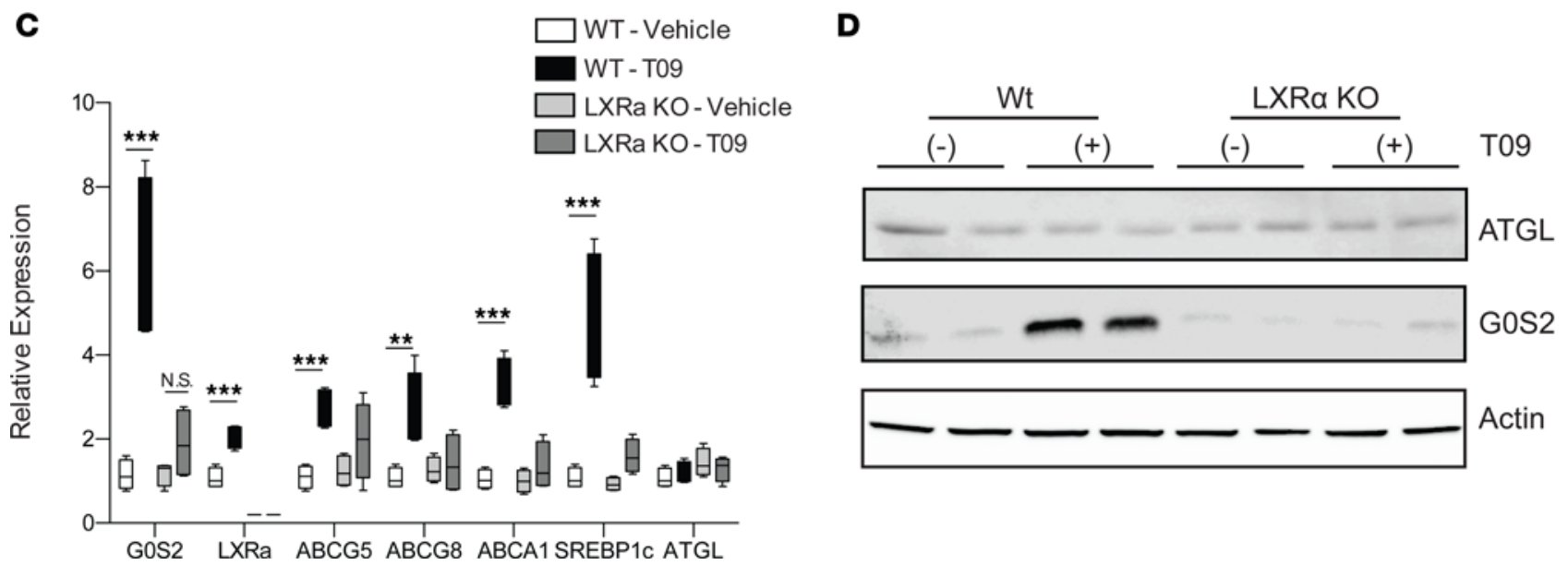

Figure 4. Activation of LXR $\alpha$ drives G0S2 transcription. (A) Ten-week-old female WT mice were injected with $20 \mathrm{mg} / \mathrm{kg} / \mathrm{day}$ of T0901317 (T09) in the presence or absence of the LXR antagonist GSK2033 or RXR $\alpha$ antagonist HX531 at concentrations described in the Methods section. qPCR analysis of COS2, ATCL, and LXR $\alpha$ target genes in liver is shown. (B) Twelve-week-old female GOS2 KO mice were injected with vehicle or TO9 as described above. Liver sections were then stained for $\beta$-galactosidase, indicating activation of the GOS2 promoter. (C) Fourteen-week-old female LXR $\alpha$ KO mice were injected with TO9 as described above. QPCR analysis of GOS2, ATCL, and LXR $\alpha$ target genes in liver was conducted. (D) Immunoblot analysis of GOS2 and ATCL expression in liver. Box-and-whisker plots depict median (line within box), 25th percentile and 75th percentile (bottom and top borders), and range of minimum to maximum values (whiskers; $n=5$ /treatment for qPCR performed in triplicate; ${ }^{*} P<0.05,{ }^{* *} P<0.01,{ }^{* * *} P<0.001$, Student's $t$ test).

promoted enrichment of LXR $\beta$ at the LXRE sites of SREBP1c and ChREBP (Figure 5D). Though a modest basal interaction was observed, the enrichment of LXR $\beta$ at the GOS2 LXRE was not further enhanced by T09 (Figure 5D). Under all 3 conditions, no significant binding signal was detected when nonspecific control IgG was used (Figure 5, B-D).

To ascertain the binding between the GOS2 LXRE sequence and LXR $\alpha /$ RXR heterodimer, we performed an EMSA using in vitro translated proteins. As shown in Figure 5E, addition of LXR $\alpha$ and RXR $\alpha$ together but not individually produced a shift complex with a biotin-labeled oligonucleotide spanning the LXRE of G0S2. An excess of unlabeled GOS2 LXRE was able to compete for complex formation, confirming the specific binding of LXR $\alpha / R X R \alpha$ to G0S2 LXRE. Binding between the G0S2 LXRE and LXR $\beta$ / $\mathrm{RXR} \alpha$ did also occur but at a slightly lower affinity (Figure $5 \mathrm{E}$ ).

To test if LXR $\alpha$ binding to the G0S2 promoter could functionally drive transcription, we constructed pGL4 luciferase reporter plasmids with varying lengths of the murine GOS2 promoter fragments encompassing the LXRE/DR4 motif (Figure 5F). When coexpressed with LXR $\alpha$ and RXR $\alpha$, both the long $(-3,010$ to -10$)$ and the short $(-2,283$ to $-1,783)$ promoter constructs gave rise to significant basal luciferase activity, which was further augmented upon the addition of T09 (Figure 5G). When the DR4 motif is specifically deleted from the short promoter fragment $(-2,283 \Delta \mathrm{DR} 4-1783)$, there was an approximate $90 \%$ reduction in the luciferase activity detected under both basal and T09-stimulated conditions (Figure 5G). Moreover, 
A

$$
\begin{array}{ll}
\text { Mouse G0S2 } & 5^{\prime}-\text { catcTGGCCTgtggTTACCTtgat }-3^{\prime} \\
\text { Rat G0S2 } & 5^{\prime}-\text { tattTGGCCCctggTCACCTtcac - 3' } \\
\text { Human G0S2 } & 5^{\prime} \text { - } \text { - ggttTGGCCCgtggTTACCTtgat - 3' }
\end{array}
$$

B

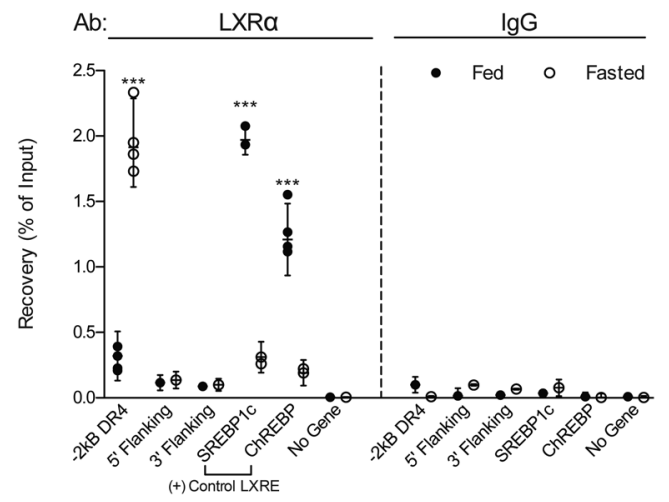

C

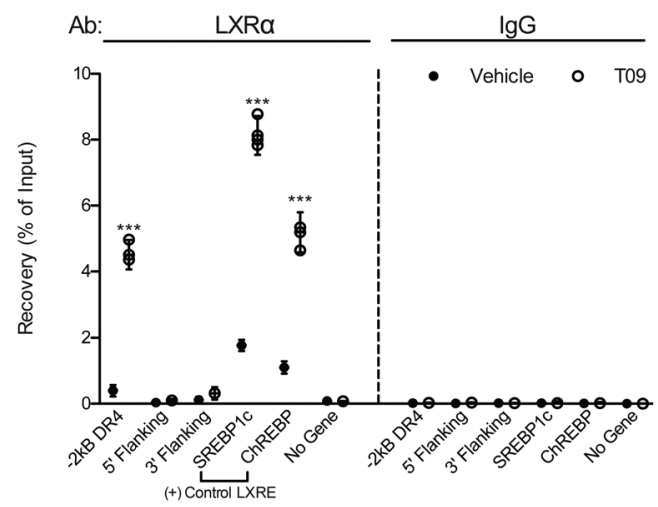

D

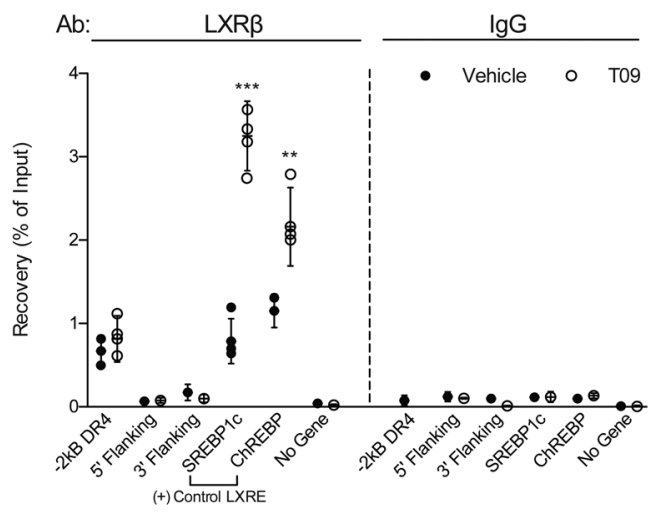

E

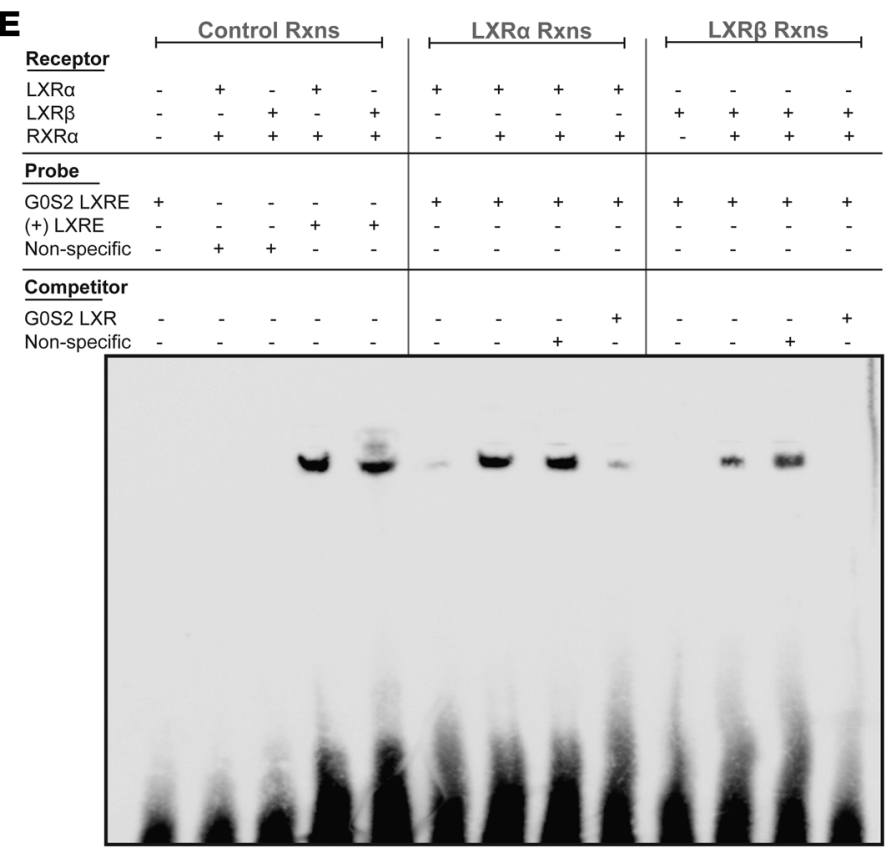

$F$

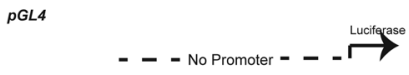

GOS2 (-2283 -1783)

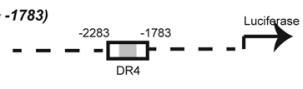

GOS2 (-3010 -10)

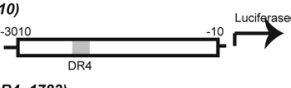
GOS2 (-2283 $\triangle D R 4$-1783)

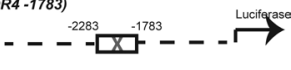

G

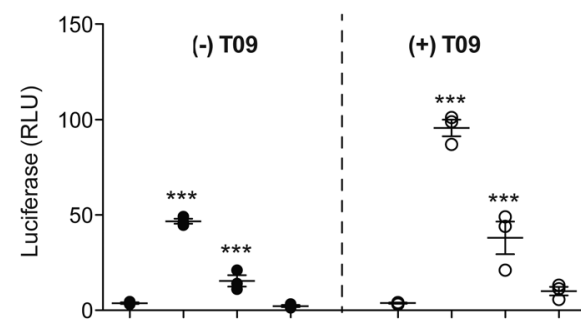

(-) LXRa/RXRa
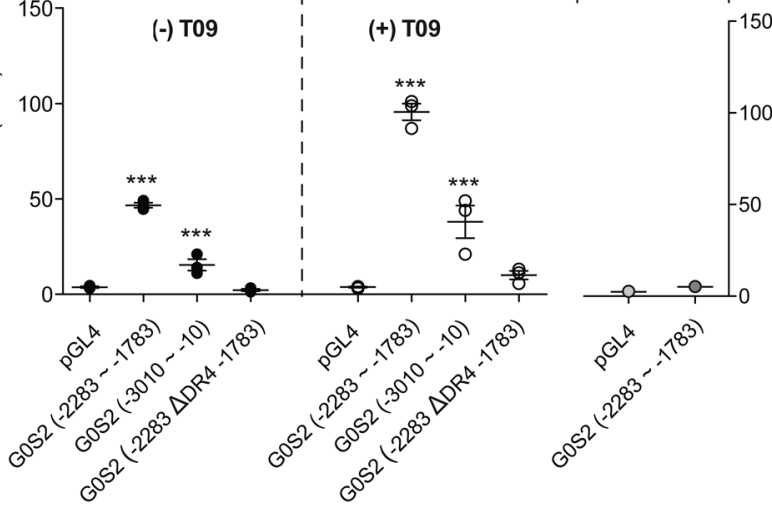

H

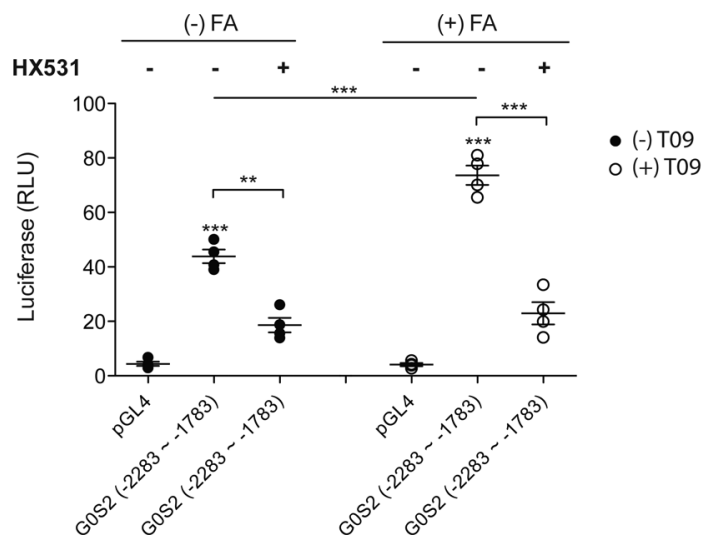

Figure 5. LXR $\alpha$ regulates GOS2 expression through direct promoter binding. (A) Sequence alignment of the putative DR4/LXRE identified in the proximal COS2 promoter across species. Twelve-week-old female WT mice were either fasted for 16 hours or injected with vehicle or T0901317 as described previously. Hepatic lysate was then subjected to ChIP. (B) QPCR analysis of LXR $\alpha$-specific ChIP chromatin from fed and fasted mice. (C) qPCR analysis of LXR $\alpha$-specific ChIP chromatin from T09-injected mice. (D) qPCR analysis of LXR $\beta$-specific ChIP chromatin from T09-injected mice. For panels B-D, primer 
sets against the putative GOS2 -2 kbDR4 LXRE, the GOS2 5' and 3' flanking regions, positive control LXREs, and a negative control were used. Data is represented as \% of input of fed vs. fasted or vehicle vs. T09. (E) EMSA assays were performed for GOS2 LXRE binding to LXR $\alpha$ and LXR $\beta$ in the presence or absence of RXR $\alpha$. Specific and nonspecific competitors were used as shown. (F) Schematic showing the promoter regions of GoS2 cloned into the pGL4 luciferase reporter system. Cotransfection assays with LXR $\alpha / R X R \alpha$ in the presence or absence of $10 \mu \mathrm{M}$ T09 or 0.725 mM C18:2 and $5 \mu \mathrm{M}$ HX531 were conducted in HeLa cells. (G) Luciferase expression in the presence or absence of T09. (H) Luciferase expression in the presence of absence of linoleic acid (FA) and HX531. ( $n=4$ for ChIP performed in duplicate, $n=3$ for luciferase assays performed in duplicate; ${ }^{* *} P<0.01,{ }^{* *} P<0.001$, Student's $t$ test.)

treatment with linoleic acid was also able to significantly induce the luciferase activity in cells transfected with LXR $\alpha$, RXR $\alpha$, and the short G0S2 promoter fragment $(-2,283$ to $-1,783$ ) (Figure $5 \mathrm{H})$, an effect that could be largely abolished by the RXR antagonist HX531. On the other hand, neither linoleic acid nor oleic acid affected the ability of a chimeric protein consisting of the ligand-binding domain of LXR $\alpha$ fused to the DNA-binding domain of Gal4 (LXR $\alpha$-LBD/Gal4-DBD) to drive a Gal4-responsive luciferase reporter in the absence of RXR (Supplemental Figure 3). Together, these data indicate that the DR4 motif identified in the G0S2 promoter is a bona fide LXRE that confers LXR $\alpha$ responsiveness, and the effect of unsaturated FAs on G0S2 promoter activity is likely mediated through RXR but not LXR $\alpha$ of the LXR $\alpha /$ RXR dimer.

GOS2 is essential for fasting-induced hepatic TG accumulation and glycogen depletion downstream of LXR . Since loss of LXR $\alpha$ leads to decreases in both hepatic TG content and G0S2 expression during fasting, we tested whether restoring G0S2 expression would be sufficient to enhance TG accumulation in the absence of LXR $\alpha$. To this end, we injected fasted WT and LXR KO mice with a recombinant adenovirus encoding mouse G0S2 (Ad-G0S2) under the control of a CMV promoter. Compared with that of a control null virus (Ad-Null), injection of Ad-G0S2 increased the hepatic G0S2 protein levels in the WT and LXR KO mice to comparable levels (Figure 6A). In Ad-Null-injected animals, LXR $\alpha$ ablation led to a pronounced reduction in the fasting hepatic TG content (Figure 6B). Ectopic expression mediated by Ad-G0S2 increased the TG content in the LXR KO mice by about 3-fold, though not to the levels observed in the WT animals (Figure 6B). These results suggest that, while multiple LXR $\alpha$-dependent factors contribute to hepatic TG accumulation during fasting, G0S2 is a key player in the process since restoring G0S2 expression alone in the absence of LXR $\alpha$ is sufficient to prominently enhance hepatic TG levels.

We also compared the changes in the adaptive energy response in fasted WT and LXR KO mice with or without Ad-G0S2 expression. To determine whether altered FA oxidation contributes to the changes in hepatic TG content, we measured the plasma levels of the ketone body 3-hydroxybutyrate (3-HB). In Ad-null-injected mice, LXR $\alpha$ ablation increased the fasting plasma levels of 3-HB by $25 \%$ (Figure 6C). Interestingly, Ad-G0S2 expression caused a $29 \%$ and $42 \%$ reduction in the plasma $3-\mathrm{HB}$ levels in the WT and LXR KO mice, respectively, resulting in no significant difference between the 2 genotypes (Figure 6C). In accordance with the previous observation, LXR $\alpha$ ablation caused a pronounced delay in the fastinginduced hepatic glycogen depletion (32), which was largely reversed with Ad-G0S2 expression (Figure 6D). Therefore, restoration of hepatic G0S2 expression in the LXR KO mice is sufficient to reverse the impaired response to fasting in terms of hepatic TG accumulation, FA oxidation, and glycogen depletion. In contrast, global energy and cholesterol metabolism appeared unaffected by LXR $\alpha$ or G0S2 since no changes were observed with plasma levels of glucose, free FA, and TG, as well as hepatic or plasma cholesterol levels in mice of all 4 groups (Figure 6E and Table 1).

GOS2 ablation prevents hepatic steatosis and hypertriglyceridemia induced by LXR agonism. To explore a possible role of GOS2 in the steatotic development induced by LXR activation, we first examined hepatic TG content in WT and G0S2 KO mice injected with T09. In the WT mice, T09 increased the fed liver and plasma TG content by $212 \%$ and $54 \%$, respectively (Figure 7, A and C). However, these effects were completely absent in the G0S2 ${ }^{-/-}$animals (Figure 7, A and C). Histological evaluation of liver specimens also revealed a lack of increased fat vacuoles in G0S2 ${ }^{-/-}$mice (Figure 7B). G0S2 ablation also led to increased plasma 3-HB levels in both vehicle- and T09-treated mice, indicating an increased hepatic FA oxidation (Supplemental Figure 4A). In addition, no significant changes were observed with plasma free FA in both groups (Supplemental Figure 4, B and C), suggesting that, regardless of G0S2, T09 treatment elicits no impact on adipose lipolysis.

Since LXR activation promotes cholesterol efflux from tissues and reverse cholesterol trafficking through HDL, we also evaluated the effects of G0S2 deletion on hepatic and plasma cholesterol profiles. In the WT mice, T09 treatment resulted in a 35\% decrease of total hepatic cholesterol (Figure 7D), a 31\% increase of total plasma cholesterol (Figure 7E), and a 36\% increase of plasma HDL levels (Figure 7F). Interestingly, 
A

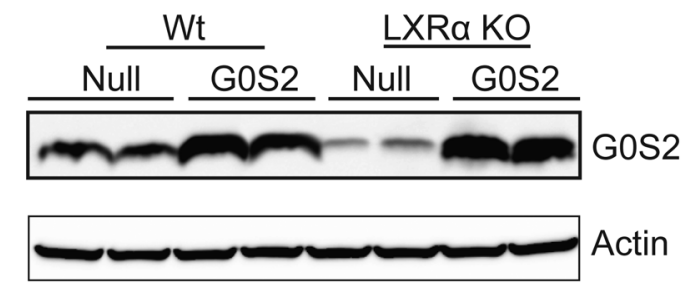

B

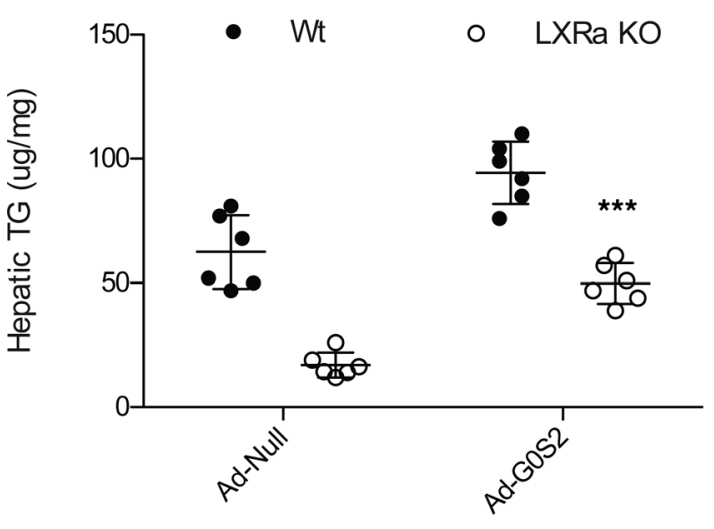

Figure 6. Adenoviral expression of G0S2 in liver of $\mathrm{LXR}^{-/-}$mice during fasting promotes lipid accumulation and glycogen depletion. Eleven-week-old female WT and LXR $\alpha$ KO mice were injected with either Ad-null or Ad-GOS2 adenovirus and then fasted for 16 hours. (A) Immunoblot analysis of GOS2 expression following adenoviral exposure. (B) Total hepatic triglyceride content, (C) plasma 3-hydroxybutyrate, (D) total hepatic glycogen content, and (E) total hepatic cholesterol content. (For TC, cholesterol, and glycogen analysis, $n=6$ for virus injected mice; ${ }^{* *} P<0.001,{ }^{*} P<$ 0.05 compared with WT, Student's $t$ test).
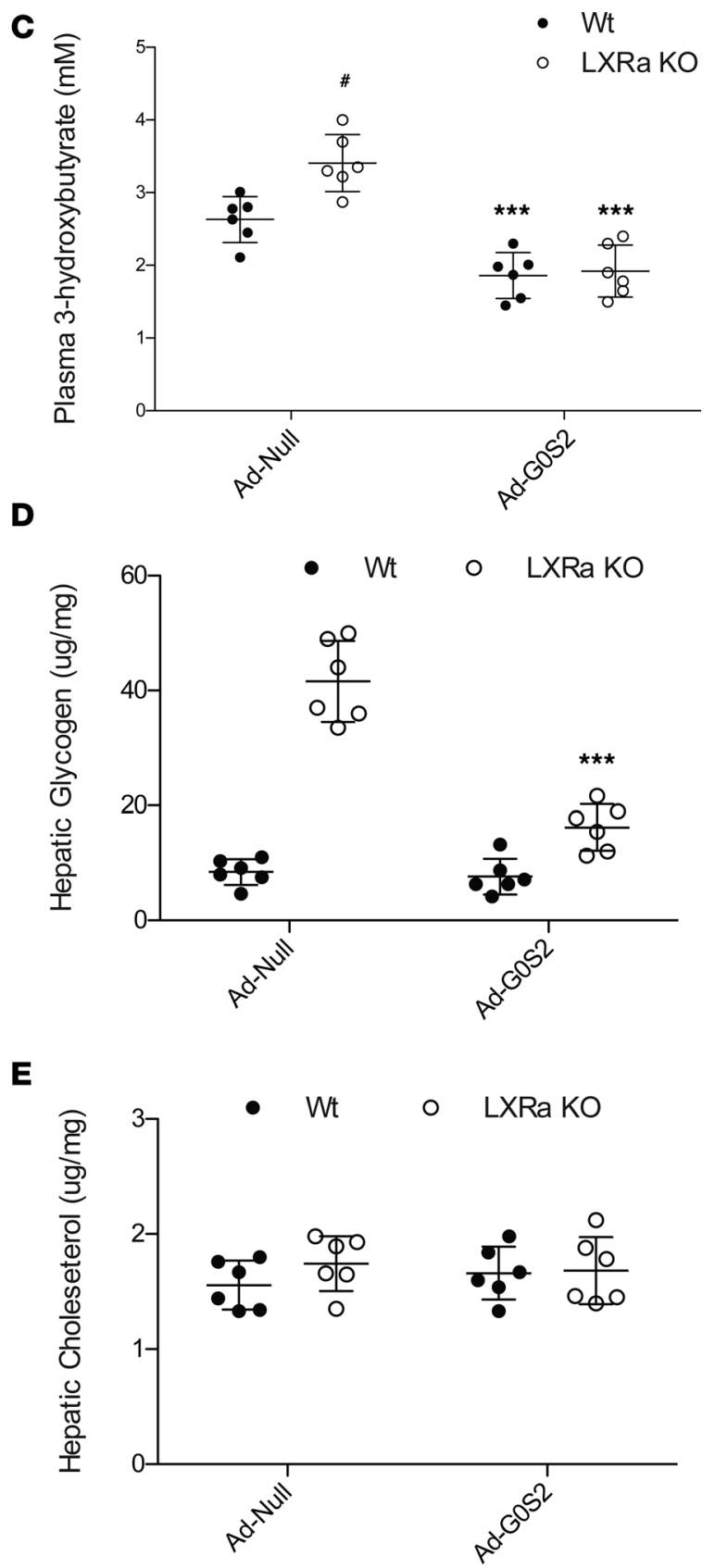

G0S2 deficiency elicited no impact on any of these T09-induced effects (Figure 7, C-G). Importantly, loss of G0S2 also did not affect the upregulation of established LXR target genes such as ABCG5, ABCG8, ABCA1, SREBP1c, and FAS by T09 (Figure 7H). The results suggest that the absence of G0S2 leads to reduced hepatic and plasma TG accumulation in the T09-treated mice; this reduction may not be caused by decreased de novo FA synthesi, and it may not be secondary to alterations in cholesterol metabolism.

\section{Discussion}

Thus far, G0S2 has been best characterized in its antilipolytic role as an inhibitor of ATGL. Recent results obtained from G0S2-deficient mice have brought us closer to a proof of concept regarding the interplay between ATGL and G0S2 in the regulation of hepatic TG mobilization and adaptive energy response to fasting (20). However, the mechanism that regulates liver G0S2 expression is still elusive. In this context, the present study provides compelling evidence that hepatic G0S2, whose expression is responsive to an influx of adipose-derived FAs during fasting, is a direct target gene of LXR $\alpha$. LXR $\alpha$ has been known to function critically in mediating fasting- and agonist-induced hepatic TG accumulation (32). In this regard, we demonstrate 
A

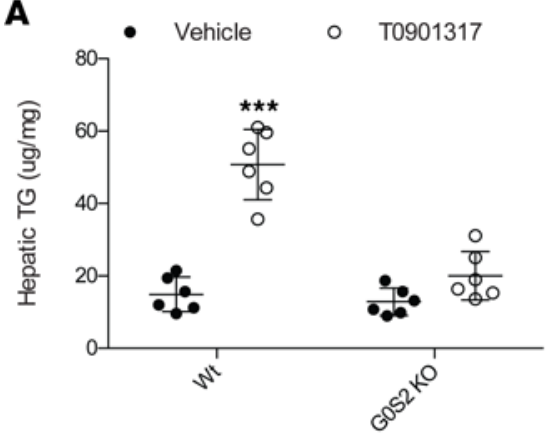

C
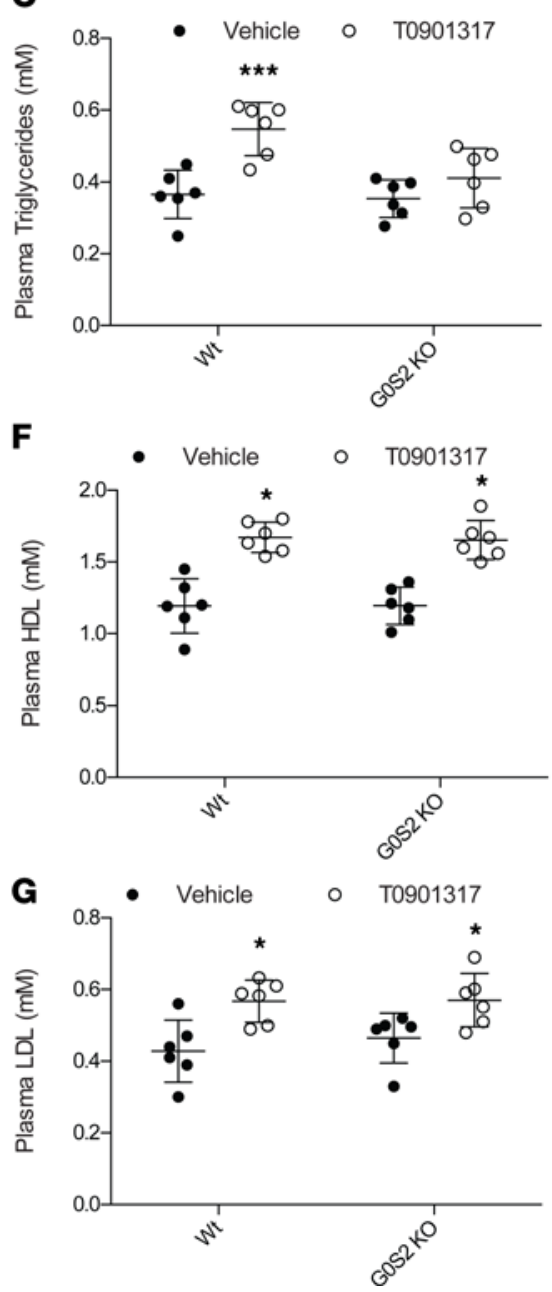

B

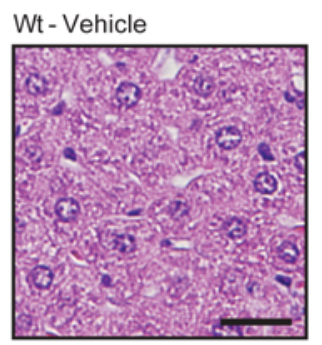

Wt - T09

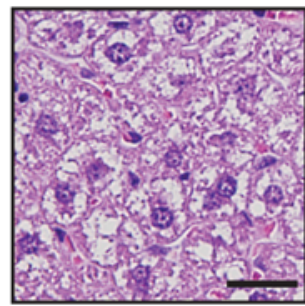

G0S2 KO - TO9

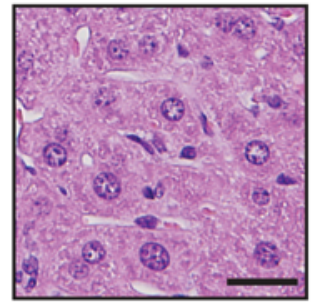

D

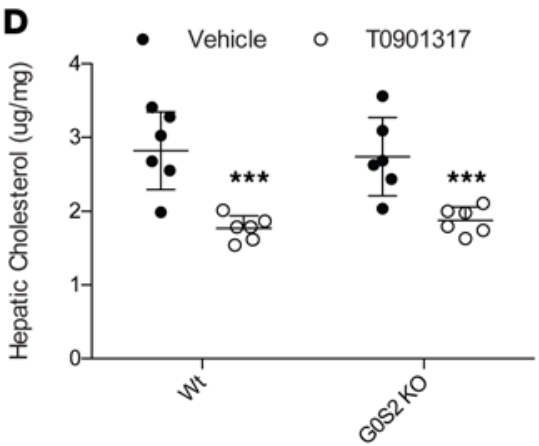

E

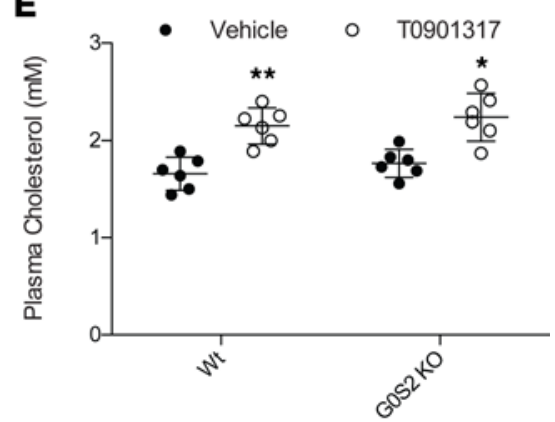

H

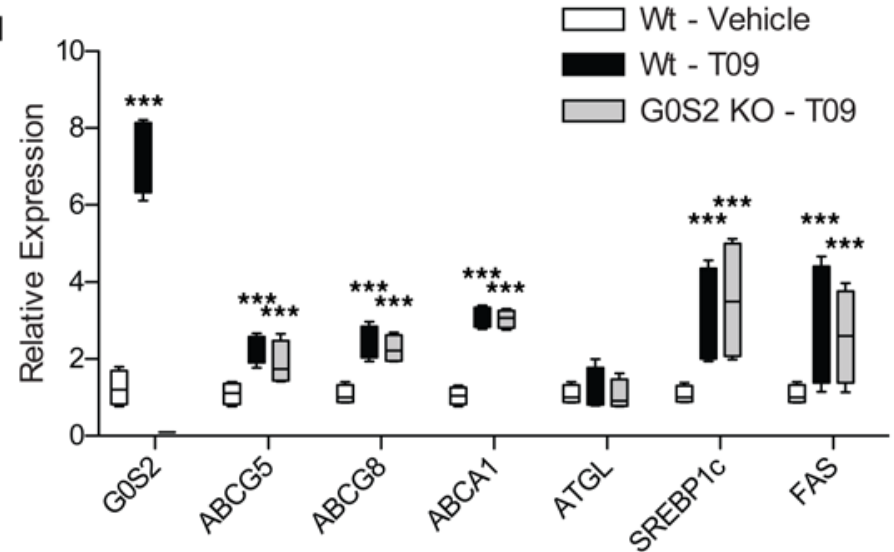

Figure 7. Hepatic steatosis and hypertriglyceridemia in response to LXR $\alpha$ activation are dependent on G0S2. Twelve-week-old female WT and G0S2 KO mice were injected with vehicle or TO9 as described previously. (A) Total hepatic triglyceride content. (B) H\&E staining of liver sections. Scale bars: $20 \mu \mathrm{m}$. (C) Plasma triglycerides (TC), (D) total hepatic cholesterol, (E) plasma cholesterol, (F) plasma HDL, and (C) plasma LDL. (H) qPCR analysis of hepatic GOS2, ATCL, LXR $\alpha$ target genes, and prolipogeneic genes. (For TC, cholesterol, glycogen, and plasma analysis, $n=6$ per group; boxand-whisker plots depict median (line within box), 25th percentile and 75th percentile (bottom and top borders), and range of minimum to maximum values (whiskers); $n=5$ for qPCR performed in triplicate; ${ }^{*} P<0.05,{ }^{* *} P<0.01,{ }^{* *} P<0.001$, Student's $t$ test).

that the LXR $\alpha$-G0S2 axis plays a pivotal role in the regulation of hepatic FA deposition in TGs under both conditions. Our results offer a new mechanism by which LXR $\alpha$ promotes liver steatosis that is separate from its established function to mediate de novo FA synthesis and reverse cholesterol trafficking.

Increased availability of adipose-derived FAs is generally believed to be the driving force for the fasting induction of hepatic adaptive energy response. These FAs not only serve as substrates for hepatic $\beta$-oxidation and TG synthesis, but also play a critical role in the regulation of hepatic gene expression. In agreement with recent observations made in CGI-58-ATko mice (23), our study using AAKO mice similarly deficient in adipose lipolysis shows that hepatic G0S2 expression is causatively linked to adipose lipolysis upon fasting. The coculture experiments further demonstrate that the effect is the consequence of a direct induction of G0S2 gene transcription in hepatocytes by unsaturated FAs, including oleic acid and linoleic acid, that are released from lipolytically stimulated adipose tissue. 
Table 1. Plasma parameters in virus injected WT and LXR $\alpha$ KO mice after 16-hour fasting

\begin{tabular}{|c|c|c|c|c|}
\hline \multirow[t]{2}{*}{ Parameter } & \multicolumn{2}{|c|}{ WT } & \multicolumn{2}{|c|}{ LXRa KO } \\
\hline & Ad-Null & Ad-GOS2 & Ad-Null & Ad-G0S2 \\
\hline Glucose (mM) & $2.37 \pm 0.31$ & $2.17 \pm 0.24$ & $2.69 \pm 0.27$ & $2.44 \pm 0.29$ \\
\hline FFA (mM) & $1.12 \pm 0.19$ & $1.19 \pm 0.15$ & $1.39 \pm 0.21$ & $1.41 \pm 0.32$ \\
\hline Total Cholesterol (mM) & $1.95 \pm 0.18$ & $1.84 \pm 0.31$ & $1.77 \pm 0.2$ & $1.69 \pm 0.17$ \\
\hline $\mathrm{HDL}(\mathrm{mM})$ & $1.24 \pm 0.19$ & $1.19 \pm 0.20$ & $1.16 \pm 0.21$ & $1.13 \pm 0.22$ \\
\hline
\end{tabular}

Eleven-week-old female WT and LXR $\alpha$ KO mice were injected with either Ad-null or Ad-GOS2 adenovirus and then fasted for 16 hours as shown in Figure 6. Blood was collected after fasting and plasma parameters were assayed. TG, triglyceride; FFA, free fatty acids; HDL, high density lipoprotein; LDL, low density lipoprotein; vLDL, very low density lipoprotein.

Using ChIP analysis on cross-linked chromatin isolated from liver tissues of fasted or agonist-treated mice, it became evident that LXR $\alpha$ but not LXR $\beta$ is abundantly enriched at the G0S2 promoter. Deletion analysis combined with a cell reporter assay confirms that the single DR4 motif within the putative LXRE of the GOS2 promoter is sufficient and required for activation by the LXR $\alpha / R X R \alpha$ heterodimer. The slightly reduced binding affinity of LXR $\beta$ for the G0S2 LXRE as revealed by the EMSA assay may in part explain the lower level of LXR $\beta$ recruitment to the G0S2 promoter as observed from the ChIP assay. It is also possible that lower expression of LXR $\beta$ relative to that of LXR $\alpha$ contributes to the lower level of interaction of the G0S2 LXRE with LXR $\beta$ detected in vivo. In this regard, LXR $\alpha$ is known to be the predominant LXR isotype in liver.

The importance of LXR $\alpha$ for GOS2 expression is recognized in experiments using LXR $\alpha \mathrm{KO}$ mice. In these mice, induction of hepatic G0S2 expression by fasting, acute stimulation of adipose lipolysis by CL, or LXR agonism by T09 was all abolished, correlating with alleviated steatosis in each case. While, in the fed state, LXRs are known to interact with insulin in the control of hepatic SREBP-1c and FA synthesis genes (38), the mechanisms that regulate LXR $\alpha$ during fasting are currently less well defined. In fasting liver, it is possible that the absence of insulin and the presence of unsaturated FAs alter the composition of cofactors and lipid mediators, thereby decreasing the binding LXR $\alpha$ to the SREBP-1c promoter while enhancing the specificity of LXR $\alpha$ for the G0S2 promoter. The finding that unsaturated FAs derived from adipose lipolysis were sufficient to induce G0S2 expression in mouse primary hepatocytes in a LXR $\alpha$ dependent manner is supportive of such a scenario. Since the RXR antagonist HX531 was able to block the effect of LXR $\alpha: R X R \alpha$ on G0S2 promoter activity in vitro as well as the hepatic expression of G0S2 in mice during fasting or acute stimulation of adipose lipolysis, we speculate that the FA responsiveness of LXR $\alpha: R X R$ is mediated via FA modification of RXR but not LXR $\alpha$. In this regard, RXR has long been recognized as an FA receptor that can be activated upon FA binding (39-43). Another observation made during this study is that hepatic LXR $\alpha$ mRNA levels in mice were increased at 16 hours of fasting. This is consistent with the findings obtained previously from fasted rats and chickens $(44,45)$. Given that LXRo expression in hepatocytes could be robustly induced by exogenously added FAs (45), it is possible that, during prolonged fasting, G0S2 expression in liver is activated by the influxed adipose-derived FAs at least in part through upregulation of LXR $\alpha$ expression.

A previous study has shown a differential expression of G0S2 in the livers of WT and PPAR $\alpha$ KO mice (37). However, it seemed unconceivable that G0S2 would be a PPAR $\alpha$ target, given the opposite roles of PPAR $\alpha$ and G0S2 in regulating hepatic FA oxidation and TG deposition during fasting $(20,21,46)$. The present study provides strong evidence that argues against a possible role of PPAR $\alpha$ in mediating G0S2 expression. For example, PPAR $\alpha$ activation by the synthetic ligand Wy14643 was insufficient to induce G0S2 expression in primary hepatocytes. Additionally, chemical antagonism of PPAR $\alpha$ in mice had no effect on fasting-induced hepatic G0S2 expression, suggesting that PPAR $\alpha$ is not required in vivo. Our findings are in agreement with the results from a previous study showing that PPAR $\alpha$ was unable to activate G0S2 expression in a direct cell reporter assay using a G0S2 promoter construct (37). A separate observation that Wy14643 failed to increase hepatic G0S2 expression in CGI-58-ATko mice also is unsupportive of a sufficient role of PPAR $\alpha$ in regulating G0S2 transcription in the absence of adipose lipolysis (23). 
It is intriguing to speculate on the in vivo significance of hepatic G0S2 induction by LXR $\alpha$. Existing evidence indicates that glucose and insulin signaling pathways are coordinated in carbohydrate feeding to synergistically activate LXRs in the liver (47-49). The induction of LXRs should lead to enhanced lipogenesis and cause an increase in newly generated FAs, providing a source of acyl residues to produce TGs through the glycerol phosphate pathway. The present study adds to this model by demonstrating that, by mediating G0S2 expression and thus attenuating ATGL-mediated lipolysis during physiological fasting, LXR $\alpha$ functions to promote the net deposition of influxed FAs in TGs, thereby limiting the amount of free FAs available for utilization in hepatocytes (Supplemental Figure 5). This could be especially relevant during the early stages of fasting when glycogen-derived glucose is the primary source of rapid energy output. Indeed, ablation of LXR $\alpha$ or G0S2 alone both led to delayed fasting-induced glycogen depletion in the liver, and hepatic overexpression of G0S2 in LXR KO mice was sufficient to accelerate glycogen breakdown while increasing TG accumulation. Moreover, we hypothesize that the increased hepatic TG storage capacity conferred by the activation of the LXR $\alpha$-G0S2 axis serves as a defense mechanism against free FA-induced lipotoxicity when the rate of FA influx exceeds the rates for FAs to be utilized for $\beta$-oxidation and VLDL synthesis. Storing TGs in the liver when FAs are in excess could also favor long-term survival during times when food deprivation is extended and the adipose depot is exhausted.

Furthermore, our evidence demonstrates a required role for G0S2 in the hepatic TG accumulation induced by the pharmacological activation of LXRs. The further finding that loss of G0S2 affected TG content but not lipogenic gene expression in response to T09 suggests that, without G0S2, increased de novo FA synthesis may be insufficient to drive TG accumulation. More importantly, synthetic LXR agonists are well documented as putative pharmacological agents for hypercholesterolemia and atherosclerosis (50-56). The LXR agonism leads to activation of the reverse pathway for cholesterol transport and both biliary and fecal cholesterol excretion. Despite their antiatherogenic benefits, the LXR agonists such as T09 also cause undesirable effects, including hypertriglyceridemia and hepatic steatosis (33, 50-56). In the present study, we demonstrate that, in the absence of G0S2, T09 is able to retain its positive effect on cholesterol/reverse cholesterol trafficking, while rendered incapable of inducing the negative side effects of increasing plasma TG and causing hepatic steatosis. Thus, our observations may open a new therapeutic strategy based on the modulation of hepatic G0S2 to alleviate hepatic steatosis and hypertriglyceridemia in response to LXR agonism.

\section{Methods}

Supplemental Methods are available online with this article.

Animal experiments. Mice lacking ATGL were generated previously by targeted homologous recombination as described (57). Heterozygous $\mathrm{ATGL}^{+/-}$mice were used to generate the whole-body ATGL KO. These mice were subsequently used for adipose tissue excision and explant culture. Adipose-specific ATGL KO mice (AAKO) were produced as previously described (36). For experimental analysis, male AAKO mice and age-matched WT littermates at 24 weeks of age were used. LXR KO mice were purchased from The Jackson Laboratory along with age-matched WT mice on C57BL6/J background. Unless otherwise noted, female mice at 10 weeks of age were used for experimental analysis. G0S2 KO mice were produced as previously reported (20). For experimental analysis, female WT and G0S2 KO littermates 12 weeks of age were used. All mice were given free access to water and were fed a standard chow diet (TestDiet, number 5001).

Agonist and antagonist injections. LXR $\alpha$ agonist T09 and GW3965, LXR $\alpha$ antagonist GSK2033, RXR $\alpha$ antagonist HX-531, PPAR $\alpha$ agonist Wy14643, and PPAR $\alpha$ antagonist GW6471 were suspended in PBS containing $0.5 \%$ Tween- 80 , which was also used as the control vehicle. Mice were injected i.p. with either drug or vehicle at a dose of $20 \mathrm{mg} / \mathrm{kg} /$ day (T09 and GSK2033) or $10 \mathrm{mg} / \mathrm{kg} /$ day (HX-531) for 3 days.

Adipose tissue explant and FA profiling. For adipose explant culture, gonadal adipose tissue was isolated from WT and ATGL KO mice. The fat pads were trimmed and placed in 12-well tissue culture dishes. Media containing high-glucose DMEM with 2\% FA-free BSA without serum was used. To induce adipose lipolysis ex vivo, the fat pads were treated with $10 \mu \mathrm{M}$ CL for 2 hours. After treatment, the media was collected and used for primary hepatocyte coculture experiments described below. For adipose lipolysis FA profiling, gonadal adipose tissue was again isolated from WT mice and explant cultured. Stimulation of lipolysis was achieved by treatment with $10 \mu \mathrm{M}$ CL for 2 hours. Media was subsequently collected and snap frozen in $\mathrm{LN}_{2}$. FA species profiling was accomplished by HPLC analysis completed by the Vanderbilt Hormone Assay Core facility (Nashville, Tennessee, USA). 
Primary hepatocyte isolation and coculture treatment. Mouse primary hepatocytes were isolated from WT mice as described (58). Hepatocytes were cultured on sterile collagen-coated tissue culture dishes for 24 hours prior to coculture with adipose tissue explant media (conditioned media). Media was collected from adipose explants, as described above. Hepatocytes were then exposed to the conditioned media for a period of 8 hours prior to lysis. Hepatocytes exposed to individual BSA-conjugated FAs were treated for 8 hours at varying doses, as described in the figure legends. Individual FA treatment concentration was consistent with the molar amount released during stimulation of adipose lipolysis by CL in explant culture.

ChIP. ChIP assays for LXR $\alpha$ and LXR $\beta$ were performed on fresh liver tissue isolated from WT mice in the fed or 16-hours fasted state, or injected with vehicle or T09, as described above. Mice were sacrificed by cervical dislocation, and the liver was rapidly excised, minced, and placed in $1 \%$ formaldehyde solution and fixed for 15 minutes at room temperature. To stop fixation, glycine was added to a final concentration of $0.125 \mathrm{M}$, and samples were mixed thoroughly. The tissue pellet was aggregated by centrifugation and washed with cold PBS twice. The fixed tissue was then homogenized in a dounce homogenizer in lysis buffer containing $10 \mathrm{mM}$ Tris- $\mathrm{HCl}$ ( $\mathrm{pH}$ 8), $10 \mathrm{mM} \mathrm{NaCl}, 0.2 \%$ NP-40, and cOmplete, Mini Protease Inhibitor Cocktail (all reagents from Sigma-Aldrich). Nuclei were then pelleted by centrifugation at $1,500 \mathrm{~g}$. The nuclear pellet was resuspended and homogenized by dounce in a nuclear lysis buffer containing $50 \mathrm{mM}$ Tris- $\mathrm{HCl}$ (pH 8), $10 \mathrm{mM} \mathrm{EDTA,} \mathrm{1 \%} \mathrm{SDS,}$ and protease inhibitors. Samples were then sonicated on ice using a VirSonic digital probe sonifier for 10 cycles at 15 seconds at a power setting of $25 \%$. Postsonication shearing controls were conducted to ensure sonication of chromatin to approximately $300-500 \mathrm{bp}$. For IP, $100 \mu \mathrm{g}$ total chromatin from each sample was diluted to $1 \mathrm{ml}$ in dilution buffer consisting of $1 \%$ triton X-100, $150 \mathrm{mM} \mathrm{NaCl}, 2 \mathrm{mM}$ EDTA, $20 \mathrm{mM}$ Tris-HCl (pH 8), and protease inhibitors. ChIP-grade antibodies against LXR $\alpha$ and LXR $\beta$ were purchased from Santa Cruz Biotechnology Inc. Normal rabbit IgG was used as an IP control (Abcam). Primary antibodies $(5 \mu \mathrm{g})$ were added to the diluted chromatin and incubated overnight at $4^{\circ} \mathrm{C}$ with rotation. Protein $\mathrm{A} / \mathrm{G}$ beads $(50 \mu \mathrm{l})$ purchased from Santa Cruz Biotechnology Inc. were then added to each IP and were incubated with rotation at $4^{\circ} \mathrm{C}$ for 1 hour. Beads were then washed 4 times in a buffer consisting of $0.1 \%$ SDS in dilution buffer. Beads were then washed 2 times in standard TE buffer with protease inhibitors. Beads were resuspended in $120 \mu$ of elution buffer containing $1 \% \mathrm{SDS}$ and $100 \mathrm{mM} \mathrm{NaHCO}_{3}$. To elute bound chromatin, the beads were slowly vortexed for 15 minutes at $30^{\circ} \mathrm{C}$. After elution, beads were centrifuged, and the supernatant was collected. $\mathrm{NaCl}$ was added, and samples were incubated overnight at $65^{\circ} \mathrm{C}$ to reverse cross-linking. RNase $\mathrm{A}(10 \mathrm{mg} / \mathrm{ml})$ and proteinase $\mathrm{K}(20 \mathrm{mg} / \mathrm{ml})$ were added and incubated with shaking at $45^{\circ} \mathrm{C}$ for 1 hour (Invitrogen). The DNA was then purified by phenol/chloroform/isoamyl alcohol extraction and resuspended in ultrapure water. Purified chromatin was then used for quantitative PCR (qPCR) analysis. Primers specific for the G0S2 -2 kb DR4/ LXRE were as follows FWD: 5' - AGAATAAAGCCAAGTCAGGATCCTC - 3' and REV: 5' - TTCAAATGTAAGGTTCACACTTGAG - 3'. Primers for the $5^{\prime}$ and $3^{\prime}$ flanking non-LXR binding regions of the G0S2 promoter were designed as follows: 5' flanking FWD: 5' - GCAATTTGTGTGTGCGTGTG - 3' and REV: 5' - CATATCTGTAATTCCAGCAA - 3' and 3' flanking FWD: 5' - AAAGAGCAGCAGCTGAGGGA 3' and REV: 5'- GGCTGCTGACTAGGGGAACT - 3'. Positive control LXRE primers were from murine SREBP-1c and ChREBP as was a nonspecific negative control primer reported previously $(26,59)$. qPCR data from ChIP samples is represented as fold enrichment between treatment conditions normalized to the IgG control samples.

Gel shift assays. LXR $\alpha, \mathrm{LXR} \beta$, and RXR $\alpha$ were in vitro translated using the TNT SP6 cell-free transcription/translation system (Promega) according to the manufacturer's protocol. The LightShift EMSA kit (Thermo Fisher Scientific) was used for the binding reactions according to the manufacturer's instructions. Duplexed 5' biotin labeled probes for SREBP-1c (+), control LXRE, G0S2 LXRE, and nonspecific, and unlabeled competitor oligos were synthesized by Integrated DNA Technologies (Supplemental Table 2). Reactions were subsequently resolved on a 5\% nondenaturing PAGE gel (in house) and transferred to positively charged nylon membranes. The LightShift DNA detection module (Thermo Fisher Scientific) was used for chemiluminescent analysis.

PCR cloning of cDNA and site-directed mutagenesis. The pGL4 luciferase reported plasmids were constructed from the $3 \mathrm{~kb}$ proximal promoter sequence of murine G0S2. Genomic DNA was extracted from mouse liver using the AllPrep Genomic DNA purification/extraction kit according to the manufacturer's instructions (Qiagen). Purified mouse genomic DNA was used as a template to amplify the $3 \mathrm{~kb}$ promoter region $10 \mathrm{bp}$ upstream of the G0S2 start codon using the following primers; FWD: 5'- TATTTTGTCCATAATCTACCACAAA - 3' and REV: 5' - TCTGCAGGCCCAGCCACCCACAGCC - 3'. For cloning into pGL4 
vector (Promega) to produce pGL4/G0S2 (-3,010 to -10$)$, NheI and HindIII restriction sites were engineered onto the $5^{\prime}$ ends of the FWD and REV primers, respectively. To construct pGL4/G0S2 ( -2283 to $-1783)$, the $3 \mathrm{~kb}$ promoter fragment was used as a template to amplify the shorter $500 \mathrm{bp}$ sequence using the following primers; FWD: 5' - TGCCCTCCTGTCAAGGCACACATCA - 3' and REV: 5' - AAGATTGCATCAGTGACCTTTGCAA - 3'. Again, NheI and HindIII sites were added as described for ligation into empty pGL4 plasmid. Site-directed mutagenesis was used as described above on pGL4/G0S2 ( $-2,283$ to -1783 ) to produce the DR4/LXRE deficient pGL4/G0S2 (-2283 $\triangle \mathrm{DR} 4-1,783)$. Primers for the mutagenesis reaction were as follows; FWD: 5' - AGAATAATCCTCATCTGATCACTTATGTAT - 3' and REV: 5' - ATACATAAGTGATCAGATGAGGATTATTCT - 3'. All plasmids were validated by restriction enzyme digestion and sequencing.

Luciferase reporter assays. For analyzing G0S2 promoter activity, HeLa cells were cotransfected with empty pGL4 or specific G0S2 pGL4 reporter plasmids, LXR $\alpha$ and RXR $\alpha$. Plasmid constructs for LXRo and RXR $\alpha$ were purchased from Addgene. For directly assaying the impact of FA on LXR $\alpha$, HepG2 cells were transfected with the LXR $\alpha$-LBD/Ga14-DBD fusion and MH100-Luc plasmids (gifts from Changcheng Zhou, University of Kentucky, Lexington, Kentucky, USA). Following transfection, cells were treated in the presence or absence of $10 \mu \mathrm{M}$ T09, $750 \mu \mathrm{M}$ linoleic acid, or $500 \mu \mathrm{M}$ oleic acid. Luciferase activity was then assayed using the Luciferase Assay System from Promega according to the manufacturer's instructions.

Statistics. Values are expressed as mean \pm SD. Statistical significance was evaluated by 2-tailed unpaired Student's $t$ test. Differences were considered significant at $P<0.05$. Quantitative data is presented as either Dot plots or Box-and-Whisker plots as described in each figure legend.

Study approval. All animal experiments were done in accordance with protocols approved by the Mayo Clinic Institutional Animal Care and Use Committee.

\section{Author contributions}

Conceptualization was done by BLH and JL; methodology was completed by BLH, XZ, and JL; investigation was performed by BLH, XZ, AMS, GS, and EEK; EEK and RZ provided resources; writing and editing was done by BLH, RZ, and JL; BLH and JL provided supervision; review and discussions were provided by EEK and RZ; and funding acquisition was provided by JL and EEK.

\section{Acknowledgments}

We would like to thank the Vanderbilt Hormone Assay Core facility for their expertise in lipid/FA profiling. We would also like to thank Changcheng Zhou (University of Kentucky) and Xitao Xie (Arizona State University) for critical discussions. This work was supported by research grants from the NIH (DK089178 and DK109096) to JL, NIH (DK090166) to EEK, as well as predoctoral funding to BLH from the Mayo Foundation for Medical Education \& Research.

Address correspondence to: Jun Liu, MCCRB 3-054, 13400 E. Shea Boulevard, Scottsdale, Arizona 85259, USA. Phone: 480.301.6745; E-mail: liu.jun@mayo.edu.

BLH's present address is: Department of Immunology, St. Jude Children's Research Hospital, Memphis, Tennessee, USA.

1. Cohen JC, Horton JD, Hobbs HH. Human fatty liver disease: old questions and new insights. Science. 2011;332(6037):1519-1523.

2. Malhi H, Gores GJ. Molecular mechanisms of lipotoxicity in nonalcoholic fatty liver disease. Semin Liver Dis. 2008;28(4):360-369.

3. Fuchs CD, Claudel T, Trauner M. Role of metabolic lipases and lipolytic metabolites in the pathogenesis of NAFLD. Trends Endocrinol Metab. 2014;25(11):576-585

4. Lin X, Yue P, Chen Z, Schonfeld G. Hepatic triglyceride contents are genetically determined in mice: results of a strain survey Am J Physiol Gastrointest Liver Physiol. 2005;288(6):G1179-G1189.

5. Guan HP, Goldstein JL, Brown MS, Liang G. Accelerated fatty acid oxidation in muscle averts fasting-induced hepatic steatosis in SJL/J mice. J Biol Chem. 2009;284(36):24644-24652.

6. Nishikawa S, Doi K, Nakayama H, Uetsuka K. The effect of fasting on hepatic lipid accumulation and transcriptional regulation of lipid metabolism differs between C57BL/6J and BALB/cA mice fed a high-fat diet. Toxicol Pathol. 2008;36(6):850-857.

7. Schweiger M, et al. G0/G1 switch gene-2 regulates human adipocyte lipolysis by affecting activity and localization of adipose triglyceride lipase. J Lipid Res. 2012;53(11):2307-2317.

8. Cornaciu I, et al. The minimal domain of adipose triglyceride lipase (ATGL) ranges until leucine 254 and can be activated and inhibited by CGI-58 and G0S2, respectively. PLoS One. 2011;6(10):e26349. 
9. Cerk IK, et al. A peptide derived from G0/G1 switch gene 2 acts as noncompetitive inhibitor of adipose triglyceride lipase. $J$ Biol Chem. 2014;289(47):32559-32570.

10. Yang $X$, et al. The $\mathrm{G}(0) / \mathrm{G}(1)$ switch gene 2 regulates adipose lipolysis through association with adipose triglyceride lipase. Cell Metab. 2010;11(3):194-205.

11. Girousse A, Langin D. Adipocyte lipases and lipid droplet-associated proteins: insight from transgenic mouse models. Int J Obes (Lond). 2012;36(4):581-594.

12. Zechner R, et al. FAT SIGNALS--lipases and lipolysis in lipid metabolism and signaling. Cell Metab. 2012;15(3):279-291.

13. Jenkins CM, Mancuso DJ, Yan W, Sims HF, Gibson B, Gross RW. Identification, cloning, expression, and purification of three novel human calcium-independent phospholipase A2 family members possessing triacylglycerol lipase and acylglycerol transacylase activities. J Biol Chem. 2004;279(47):48968-48975.

14. Villena JA, Roy S, Sarkadi-Nagy E, Kim KH, Sul HS. Desnutrin, an adipocyte gene encoding a novel patatin domain-containing protein, is induced by fasting and glucocorticoids: ectopic expression of desnutrin increases triglyceride hydrolysis. $J$ Biol Chem. 2004;279(45):47066-47075.

15. Zimmermann R, et al. Fat mobilization in adipose tissue is promoted by adipose triglyceride lipase. Science. 2004;306(5700):1383-1386.

16. Zechner R, Kienesberger PC, Haemmerle G, Zimmermann R, Lass A. Adipose triglyceride lipase and the lipolytic catabolism of cellular fat stores. J Lipid Res. 2009;50(1):3-21.

17. Nielsen TS, Møller N. Adipose triglyceride lipase and G0/G1 switch gene 2: approaching proof of concept. Diabetes. 2014;63(3):847-849

18. Heier C, et al. G0/G1 Switch Gene 2 Regulates Cardiac Lipolysis. J Biol Chem. 2015;290(43):26141-26150.

19. Heier C, Zimmermann R. The sparing use of fat: G0s2 controls lipolysis and fatty acid oxidation. Diabetologia. 2015;58(1):7-9.

20. Zhang X, Xie X, Heckmann BL, Saarinen AM, Czyzyk TA, Liu J. Targeted disruption of G0/G1 switch gene 2 enhances adipose lipolysis, alters hepatic energy balance, and alleviates high-fat diet-induced liver steatosis. Diabetes. 2014;63(3):934-946.

21. El-Assaad W, et al. Deletion of the gene encoding G0/G 1 switch protein 2 (G0s2) alleviates high-fat-diet-induced weight gain and insulin resistance, and promotes browning of white adipose tissue in mice. Diabetologia. 2015;58(1):149-157.

22. Ma T, et al. Mice lacking G0S2 are lean and cold-tolerant. Cancer Biol Ther. 2014;15(5):643-650.

23. Jaeger D, et al. Fasting-induced G0/G1 switch gene 2 and FGF21 expression in the liver are under regulation of adipose tissue derived fatty acids. J Hepatol. 2015;63(2):437-445.

24. Hong C, Tontonoz P. Liver X receptors in lipid metabolism: opportunities for drug discovery. Nat Rev Drug Discov. 2014;13(6):433-444

25. Ducheix S, Montagner A, Theodorou V, Ferrier L, Guillou H. The liver X receptor: a master regulator of the gut-liver axis and a target for non alcoholic fatty liver disease. Biochem Pharmacol. 2013;86(1):96-105.

26. Repa JJ, et al. Regulation of mouse sterol regulatory element-binding protein-1c gene (SREBP-1c) by oxysterol receptors, LXRalpha and LXRbeta. Genes Dev. 2000;14(22):2819-2830.

27. Yoshikawa $\mathrm{T}$, et al. Identification of liver $\mathrm{X}$ receptor-retinoid $\mathrm{X}$ receptor as an activator of the sterol regulatory element-binding protein 1c gene promoter. Mol Cell Biol. 2001;21(9):2991-3000.

28. Joseph SB, et al. Direct and indirect mechanisms for regulation of fatty acid synthase gene expression by liver X receptors. $J$ Biol Chem. 2002;277(13):11019-11025

29. Shimano H, Horton JD, Shimomura I, Hammer RE, Brown MS, Goldstein JL. Isoform 1c of sterol regulatory element binding protein is less active than isoform 1a in livers of transgenic mice and in cultured cells. J Clin Invest. 1997;99(5):846-854.

30. Kalaany NY, et al. LXRs regulate the balance between fat storage and oxidation. Cell Metab. 2005;1(4):231-244.

31. Korach-André M, et al. Liver X receptors regulate de novo lipogenesis in a tissue-specific manner in C57BL/6 female mice. Am J Physiol Endocrinol Metab. 2011;301(1):E210-E222.

32. Oosterveer MH, et al. Lxralpha deficiency hampers the hepatic adaptive response to fasting in mice. J Biol Chem. 2008;283(37):25437-25445.

33. Zhang Y, et al. Liver LXR $\alpha$ expression is crucial for whole body cholesterol homeostasis and reverse cholesterol transport in mice. J Clin Invest. 2012;122(5):1688-1699.

34. Bischoff ED, et al. Non-redundant roles for LXRalpha and LXRbeta in atherosclerosis susceptibility in low density lipoprotein receptor knockout mice. J Lipid Res. 2010;51(5):900-906.

35. Quinet EM, et al. Liver X receptor (LXR)-beta regulation in LXRalpha-deficient mice: implications for therapeutic targeting Mol Pharmacol. 2006;70(4):1340-1349.

36. Schoiswohl G, et al. Impact of Reduced ATGL-Mediated Adipocyte Lipolysis on Obesity-Associated Insulin Resistance and Inflammation in Male Mice. Endocrinology. 2015;156(10):3610-3624.

37. Zandbergen F, et al. The G0/G1 switch gene 2 is a novel PPAR target gene. Biochem J. 2005;392(Pt 2):313-324.

38. Sun X, et al. Insulin Dissociates the Effects of Liver X Receptor on Lipogenesis, Endoplasmic Reticulum Stress, and Inflammation. J Biol Chem. 2016;291(3):1115-1122.

39. Hong C, Tontonoz P. Liver X receptors in lipid metabolism: opportunities for drug discovery. Nat Rev Drug Discov. 2014;13(6):433-444

40. Lengqvist $\mathrm{J}$, et al. Polyunsaturated fatty acids including docosahexaenoic and arachidonic acid bind to the retinoid $\mathrm{X}$ receptor alpha ligand-binding domain. Mol Cell Proteomics. 2004;3(7):692-703.

41. Nakamura MT, Yudell BE, Loor JJ. Regulation of energy metabolism by long-chain fatty acids. Prog Lipid Res. 2014;53:124-144.

42. Steineger HH, Arntsen BM, Spydevold O, Sørensen HN. Gene transcription of the retinoid X receptor alpha (RXRalpha) is regulated by fatty acids and hormones in rat hepatic cells. J Lipid Res. 1998;39(4):744-754.

43. Fan YY, Spencer TE, Wang N, Moyer MP, Chapkin RS. Chemopreventive n-3 fatty acids activate RXRalpha in colonocytes. Carcinogenesis. 2003;24(9):1541-1548.

44. Désert C, et al. Transcriptome profiling of the feeding-to-fasting transition in chicken liver. BMC Genomics. 2008;9:611.

45. Tobin KA, et al. Cross-talk between fatty acid and cholesterol metabolism mediated by liver X receptor-alpha. Mol Endocrinol. 
2000;14(5):741-752.

46. Hashimoto T, Cook WS, Qi C, Yeldandi AV, Reddy JK, Rao MS. Defect in peroxisome proliferator-activated receptor alpha-inducible fatty acid oxidation determines the severity of hepatic steatosis in response to fasting. J Biol Chem. 2000;275(37):28918-28928.

47. Tobin KA, et al. Liver X receptors as insulin-mediating factors in fatty acid and cholesterol biosynthesis. J Biol Chem 2002;277(12):10691-10697.

48. Chen G, Liang G, Ou J, Goldstein JL, Brown MS. Central role for liver X receptor in insulin-mediated activation of Srebp-1c transcription and stimulation of fatty acid synthesis in liver. Proc Natl Acad Sci USA. 2004;101(31):11245-11250.

49. Anthonisen EH, Berven L, Holm S, Nygård M, Nebb HI, Grønning-Wang LM. Nuclear receptor liver X receptor is O-GlcNAcmodified in response to glucose. J Biol Chem. 2010;285(3):1607-1615.

50. Briand F, et al. Liver X receptor activation promotes macrophage-to-feces reverse cholesterol transport in a dyslipidemic hamster model. J Lipid Res. 2010;51(4):763-770.

51. Kannisto K, et al. LXR driven induction of HDL-cholesterol is independent of intestinal cholesterol absorption and ABCA1 protein expression. Lipids. 2014;49(1):71-83

52. Ou X, et al. Liver X receptor agonist T0901317 reduces atherosclerotic lesions in apoE-/- mice by up-regulating NPC1 expression. Sci China, C, Life Sci. 2008;51(5):418-429.

53. Plōsch T, et al. Increased hepatobiliary and fecal cholesterol excretion upon activation of the liver $\mathrm{X}$ receptor is independent of ABCA1. J Biol Chem. 2002;277(37):33870-33877.

54. Yasuda T, et al. Tissue-specific liver X receptor activation promotes macrophage reverse cholesterol transport in vivo. Arterioscler Thromb Vasc Biol. 2010;30(4):781-786.

55. Zanotti I, et al. The LXR agonist T0901317 promotes the reverse cholesterol transport from macrophages by increasing plasma efflux potential. J Lipid Res. 2008;49(5):954-960.

56. Cha JY, Repa JJ. The liver X receptor (LXR) and hepatic lipogenesis. The carbohydrate-response element-binding protein is a target gene of LXR. J Biol Chem. 2007;282(1):743-751.

57. Haemmerle G, et al. Defective lipolysis and altered energy metabolism in mice lacking adipose triglyceride lipase. Science. 2006;312(5774):734-737.

58. Chen Z, Fitzgerald RL, Averna MR, Schonfeld G. A targeted apolipoprotein B-38.9-producing mutation causes fatty livers in mice due to the reduced ability of apolipoprotein B-38.9 to transport triglycerides. J Biol Chem. 2000;275(42):32807-32815.

59. Boergesen M, et al. Genome-wide profiling of liver X receptor, retinoid X receptor, and peroxisome proliferator-activated receptor $\alpha$ in mouse liver reveals extensive sharing of binding sites. Mol Cell Biol. 2012;32(4):852-867. 